DOI: 10.1002/adsc.200((will be filled in by the editorial sttaff))

\title{
Photopromoted Entry to Benzothiophenes, Benzoselenophenes, $3 H$-Indoles, Isocoumarins, Benzosultams, and (Thio)Flavones by Gold-Catalyzed Arylative Heterocyclization of Alkynes
}

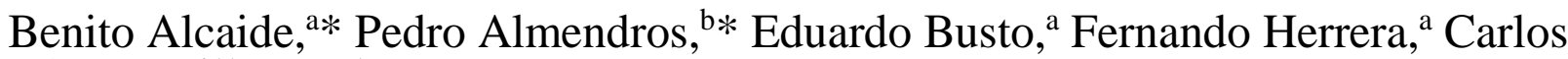 \\ Lázaro-Milla, ${ }^{a}$ and Amparo Luna ${ }^{a}$
}

a Grupo de Lactamas y Heterociclos Bioactivos, Departamento de Química Orgánica I, Unidad Asociada al CSIC, Facultad de Química, Universidad Complutense de Madrid, 28040 Madrid, Spain, Fax: (+34) 91-3944103, E-mail: alcaideb@quim.ucm.es

b Instituto de Química Orgánica General, Consejo Superior de Investigaciones Científicas, IQOG-CSIC, Juan de la Cierva 3, 28006 Madrid, Spain, Fax: (+34) 91-5644853, E-mail: Palmendros@iqog.csic.es

Received: ((will be filled in by the editorial staff))

Supporting information for this article is available on the WWW under http://dx.doi.org/10.1002/adsc.200\#\#\#\#\#.((Please delete if not appropriate))

\begin{abstract}
Visible light-promoted and gold-photoredox catalyzed reactions of heteroatom( $\mathrm{N}, \mathrm{S}$, Se, O)-tethered alkynes with arenediazonium salts selectively reacted to build vicinal diaryl-substituted $2 H$-benzo[e][1,2]thiazine 1,1dioxides (benzosultams), benzoselenophenes, benzothiophenes, $4 \mathrm{H}$-chromen-4-ones (flavones), $3 \mathrm{H}$ indoles, $1 \mathrm{H}$-isochromen-1-ones (isocoumarins), and $4 \mathrm{H}$ -
\end{abstract}

thiochromen-4-ones (thioflavones). Moreover, the utility of functionalized $3 \mathrm{H}$-indoles as precursors for further elaboration has been demonstrated with the switchable and facile preparation of $1 \mathrm{H}$-indoles, 2-oxindoles, and 3 oxindolines.

Keywords: alkynes; cyclization; gold; heterocyclic compounds; synthetic methods

\section{Introduction}

Homogeneous gold catalysis has been developed as a potent tool in the field of synthetic organic chemistry. Particularly attractive is the activation of alkenes, alkynes and allenes by cationic gold(I) species. ${ }^{[1]}$ However, $\mathrm{Au}(\mathrm{I}) / \mathrm{Au}(\mathrm{III})$ catalytic cycles cannot be accessed through the traditional gold(I)-catalyzed processes, and super-stoichiometric amounts of a strong oxidant are required for surpassing the high redox potential $\mathrm{Au}(\mathrm{I}) / \mathrm{Au}(\mathrm{III}){ }^{[2]}$ Taking into account the above limitations, it is not surprising that goldcatalyzed cross-coupling strategies are a less explored field. Glorius and later Toste developed a smart strategy for avoiding the drawback of the inclusion of strong oxidants, ${ }^{[3]}$ which takes advantage of a photoredox catalyst and a diazonium salt. ${ }^{[4]}$ Hashmi and Barriault developed gold-only photoredox chemistry through the use of dinuclear complexes of gold as photoredox catalysts. ${ }^{[5]}$

On the other hand, the widespread presence of heterocycles in both natural products and synthetic drugs as well as in advanced materials, explains the interest in the preparation of these cyclic frameworks. This area is clearly dominated by palladium catalysis, which usually demands elevated temperatures and the use of ligands and bases. Besides, the incorporation of aryl substituents to the heterocyclic core is performed by the use of aryl halides, which are not always readily available. Aiming to surmount these deficiencies, we were motivated to include diazonium salts and visible light in a comprehensive gold-catalyzed arylative heterocycle formation. Due to environmental concerns, the development of a photocatalyzed arylative synthesis at room temperature may be a great achievement. We wish to describe herein the unique use of diazonium salts as a radical source in the cooperative gold-photoredox catalyzed synthesis of benzo-fused heterocycles (Scheme 1). ${ }^{[6]}$ 


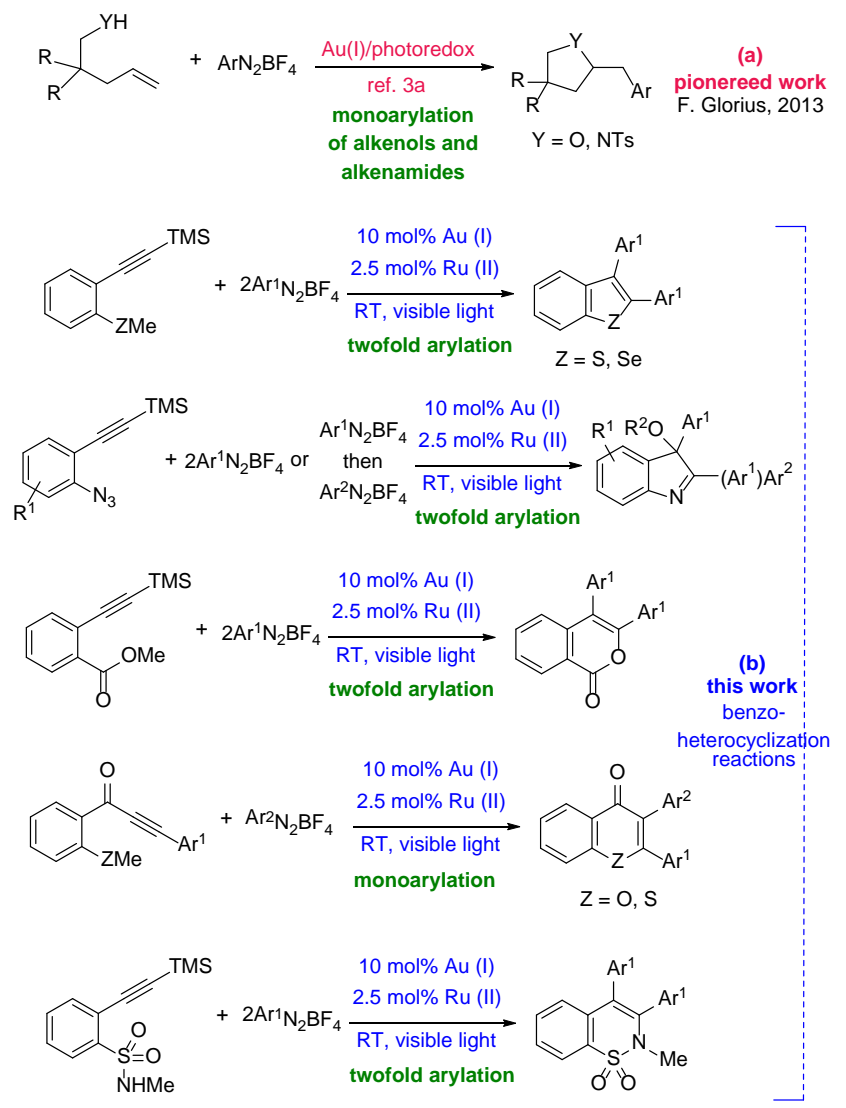

Scheme 1. Dual gold- and photoredox-catalyzed heterocyclization reactions: Previous and current strategies. TMS = Trimethylsilyl.

\section{Results and Discussion}

(2-Ethynylphenyl)(methyl)sulfane 1a-H-SMe and phenyldiazonium salt 2a were chosen as model substrates to examine their reactivity under cooperative gold and photoredox catalysis. ${ }^{[7]}$ Our aim was the in situ generation of a methyl[2(arylethynyl)phenyl]sulfane from a gold-catalyzed sila-Sonogashira-type coupling, which can be further trapped by the nucleophile sulphur with a concomitant second arylation in a domino sequence. This premise deals with difficulties and presents an initial challenge because the photoredox gold-catalyzed arylation of sulphur derivatives has not yet been reported. Besides, we need that the starting material undergoes a relatively fast coupling at the alkyne site, in comparison with the heterocyclization event. Substrate 1a-H-SMe provided benzothiophene $\mathbf{3 a - S}$ in a promising $27 \%$ yield. A significant improvement was detected with the use of TMS-capped alkyne 1a-SiSMe ${ }^{[8]}$ which was considered as viable precursor in the synthesis of 2,3-diaryl benzothiophenes. We initiated our optimization studies using various gold(I) complexes and the ruthenium salt $\left.\left[\mathrm{Ru}(\mathrm{bpy})_{3}\right]\left(\mathrm{PF}_{6}\right)_{2}\right]$ (bpy $=2,2$ '-bipyridine) as shown in Table 1 . After the evaluation of different conditions, $\mathrm{PPh}_{3} \mathrm{AuCl}(10$ mol\%) in combination with $\left.\left[\mathrm{Ru}(\mathrm{bpy})_{3}\right]\left(\mathrm{PF}_{6}\right)_{2}\right](2.5$ mol\%) provided an impressive yield (94\%) of 2,3diphenyl benzothiophene 3a-S (entry 3, Table 1).
When the loading of $\mathrm{PPh}_{3} \mathrm{AuCl}$ was increased from 10 mol\% to $20 \mathrm{~mol} \%$, it resulted in just a little improvement of the yield (95\%) (entry 5, Table 1). Decreasing the amount of $\mathrm{PPh}_{3} \mathrm{AuCl}$ from $10 \mathrm{~mol} \%$ to $5 \mathrm{~mol} \%$ produced an appreciable reduction of the yield (64\%) (entry 6, Table 1). Inferior results were obtained when the reaction was carried out in presence of other photoactive complexes such as $\left.\left[\operatorname{Ir}(\mathrm{ppy})_{2}(\mathrm{dtbbpy})\right]\left(\mathrm{PF}_{6}\right)_{2}\right]$ (ppy = 2-phenylpyridine; dtbbpy = 4,4'-di-tert-butyl-2,2'-bipyridine) (entry 4, Table 1). Also, alternative gold(I) sources such as [AuClIPr] $\quad(\mathrm{IPr}=1,3$-bis(2,6diisopropylphenyl)imidazol-2-ylidene) and $\left[\left(\mathrm{Ph}_{3} \mathrm{P}\right) \mathrm{AuNTf}_{2}\right]$ were inefficient (entries 1 and 2, Table 1).

Table 1. Screening of reaction conditions for benzothiophene formation through light-driven gold/photoredox-co-catalyzed diarylative thiacyclization. ${ }^{[a]}$

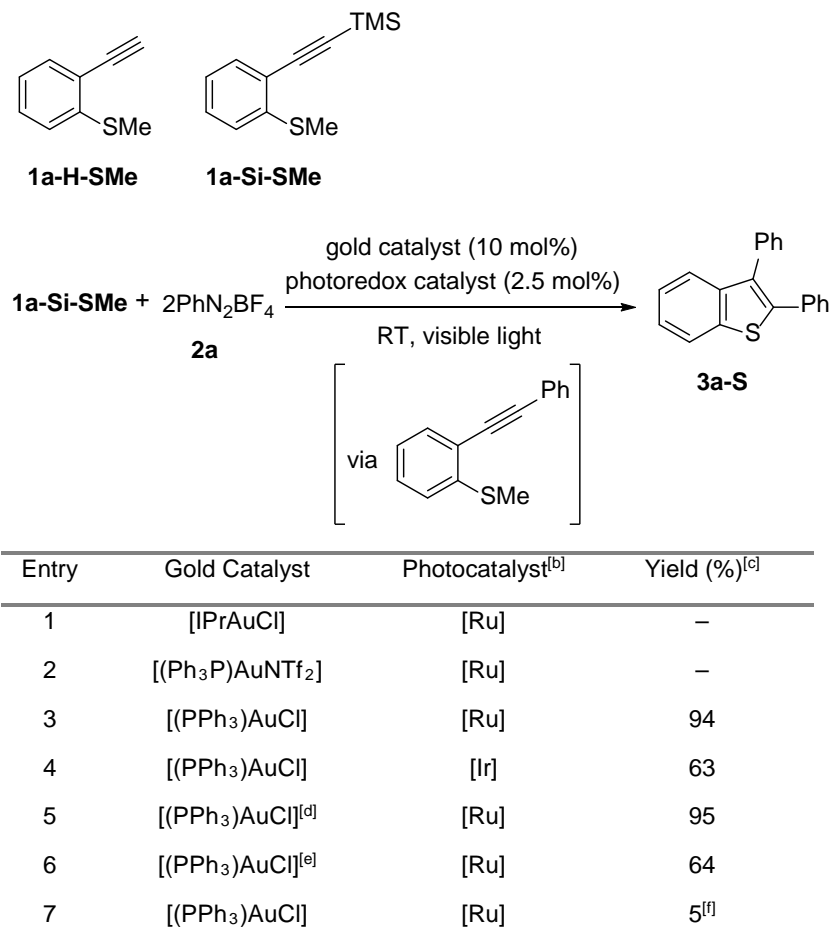

[a] Unless otherwise noted, all reactions were carried out in methanol/acetonitrile (3:1) at room temperature.

[b] $\left.[\mathrm{Ru}]=\left[\mathrm{Ru}(\mathrm{bpy})_{3}\right]\left(\mathrm{PF}_{6}\right)_{2}\right] . \quad[\mathrm{Ir}]=$ $\left[\operatorname{Ir}(\text { ppy })_{2}\right.$ (dtbbpy) $\left.]\left(\mathrm{PF}_{6}\right)_{2}\right]$.

${ }^{[c]}$ Yield of pure, isolated product with correct analytical and spectral data.

${ }^{\text {[d] }}$ Catalyst loading of $20 \mathrm{~mol} \%$.

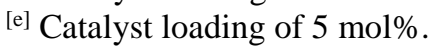

${ }^{[f]}$ The reaction was carried out in DMF.

We applied the above reaction conditions to differently functionalized arenediazonium salts 2 (Scheme 2). Substitution was tolerated in the diarylative thiacyclizations of 1a-Si-SMe, with the presence at the arenediazonium salt of electron withdrawing groups $\left(\mathrm{CO}_{2} \mathrm{Et}, \mathrm{CF}_{3}\right)$ as well as halogens $(\mathrm{Br})$ and weakly electron donating groups $(\mathrm{Me})$. The results depicted in the above Scheme show the effectiveness of this methodology for the smooth 
preparation of a variety of 2,3-diaryl benzothiophenes 3-S. ${ }^{[9]}$

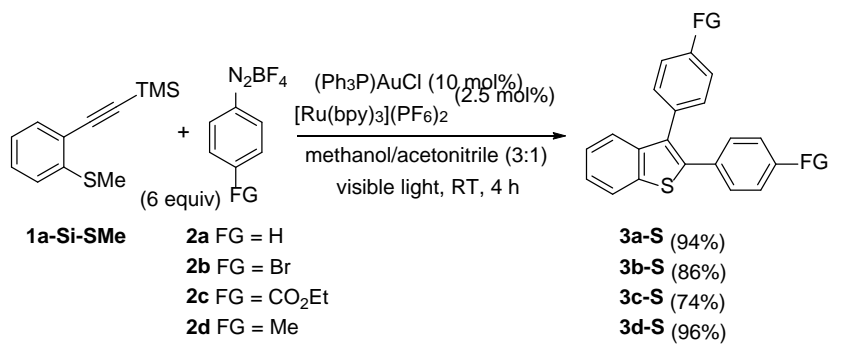

Scheme 2. Cooperative gold-photoredox catalysis for the synthesis of 2,3-diaryl benzothiophenes 3-S.

To further probe the scope and versatility of the arylative carbon-chalcogen cyclization reaction, trimethyl[(2-methylselanylphenyl)ethynyl]silane 1aSi-SeMe was used to react with phenyldiazonium salt 2a under the optimized conditions for the formation of 2,3-diaryl benzothiophenes 3-S. As depicted in Scheme 3 , the diarylation/C-Se bond formation sequence proceeded to afford 2,3-diaryl benzoselenophene 3a-Se, but less efficiently. Precursor 1a-Si-SeMe reacted well with several arenediazonium salts 2 bearing diverse substitution and gave rise under mild conditions to the desired selenoheterocycles 3-Se, a type of organic molecules which are prevalent both in drugs and in advanced materials (Scheme 3).

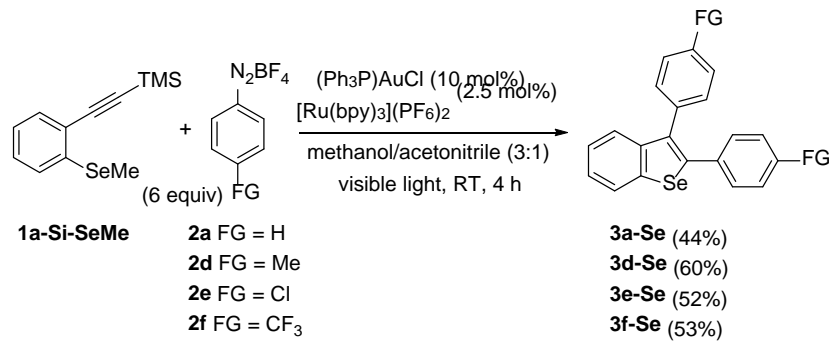

Scheme 3. Cooperative gold-photoredox catalysis for the synthesis of 2,3-diaryl benzoselenophenes 3-Se.

Continuing to explore different functionalities at the TMS-protected alkynes and taking into account that the azide moiety is a versatile nitrogen source, ${ }^{[10]}$ we decided to test azidobenzene-tethered alkynes. ${ }^{[11]}$ The above reaction conditions for TMS-sulfane 1a-SiSMe was also applicable for TMS(ethynyl)azidobenzene $\mathbf{4 a - S i}{ }^{[12]}$ but it exhibited different reactivity. The lack of formation of expected 2,3-diaryl $1 \mathrm{H}$-indoles of type $\mathbf{5}$ was observed, which should point to a marked directing effect of the heteroatomic nucleophile functionality. In view of the structure of adduct $\mathbf{6 a}$, the participation of methanol as a nucleophile is apparent. Noteworthy, this interesting reactivity switch allowed the obtention of functionalized $3 H$-indoles $\mathbf{6 a a}-\mathbf{a h}$ through a diarylative aminocyclization/hydroalkoxylation sequence (Scheme 4). Halogenated or weakly activated arenediazonium salts turned out to be suitable coupling partners. However, diazonium salts having strong electron withdrawing groups $\left(\mathrm{NO}_{2}\right.$ and
$\mathrm{CF}_{3}$ ) failed to give the desired indole. Instead, alkenes 7 were formed (Scheme 5). Apparently, the electron poor aryl alkyne intermediate disfavors the aminocyclization with the azide moiety and the competitive intermolecular nucleophilic addition of methanol dominates.

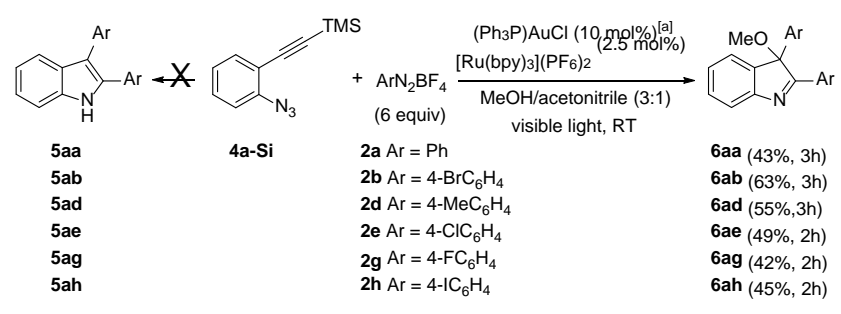

Scheme 4. Cooperative gold-photoredox catalysis for the synthesis of 2,3-diaryl $3 \mathrm{H}$-indoles 6. [a] For a full conversion, the complex was added in two separated portions with a $30 \mathrm{~min}$ spacing.

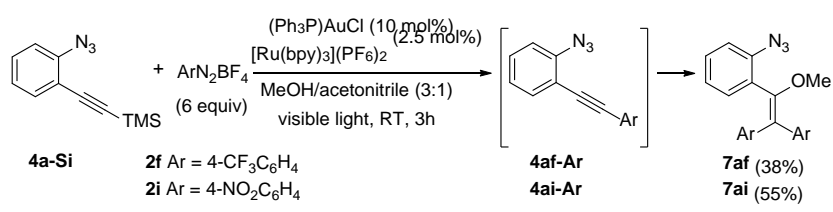

Scheme 5. Cooperative gold-photoredox catalysis for the diarylative hydroalkoxylation of (ethynyl)azidobenzene 4aSi with strongly deactivated arenediazonium salts.

Next, under the optimized reaction conditions, the reactivities of several TMS-(ethynyl)azidobenzenes 4Si with arenediazonium salt $\mathbf{2 b}$ were examined (Scheme 6). The power of this methodology was further demonstrated with the use of different alcohols (ethanol, 2-propanol, tert-butanol, and methanol- $\mathrm{d}_{3}$ ) instead of methanol to give adducts 6ab-Et, 6ab-iPr, 6ab-tBu, and 6ab-CD $\mathbf{C D}_{3}$ (Scheme 6). Besides, several substituents could be incorporated to the starting materials, which grants for the formation of a variety of functionalized $3 \mathrm{H}$-indoles. The reaction yields $\left({ }^{1} \mathrm{H}\right.$ NMR) were excellent before purification because no side-products were detected when the starting material was fully converted. However, partial decomposition was observed on sensitive $3 H$-indoles 6 during chromatographic purification.

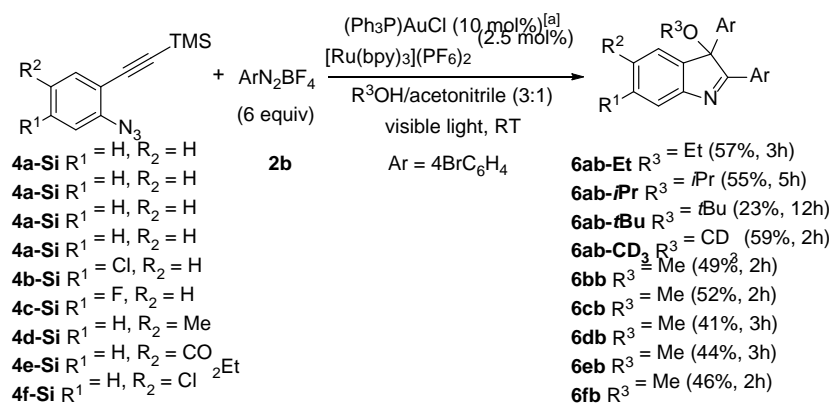

Scheme 6. Cooperative gold-photoredox catalysis for the synthesis of differently substituted 2,3-diaryl $3 \mathrm{H}$-indoles $\mathbf{6}$. [a] For a full conversion, the complex was added in two separated portions with a 30 min spacing. 
To fully exploit the potential of this methodology, we reacted [(2-azidophenyl)ethynyl]trimethylsilane 4a-Si with a pair of different diazonium salts 2 . Initial Hiyama coupling of TMS-precursor 4a-Si with the first diazonium salt $\mathbf{2}$ provided 1-azido-2(arylethynyl)benzene intermediates. Without the need of isolation, these intermediates afforded the final differently diarylated $3 \mathrm{H}$-indoles $\mathbf{6}$ after a second coupling with another diazonium salt (Scheme 7). Thus, TMS-(ethynyl)azidobenzene $\mathbf{4 a - S i}$ acts as a versatile building block for the double crossed diarylation reaction, and allows for the preparation of crossover adducts in a convenient and modular way.

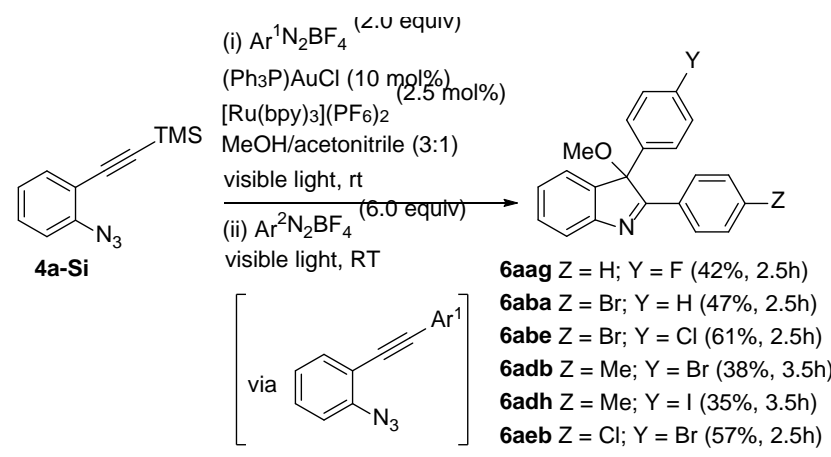

Scheme 7. Cooperative gold-photoredox catalysis for the crossed preparation of 2,3-diaryl $3 \mathrm{H}$-indoles 6.

Besides, the utility of 3-alkoxy-2,3-diaryl-3Hindoles $\mathbf{6}$ as precursors for further elaboration has been demonstrated with the controlled preparation of $\mathrm{N}$ unprotected indoles $\mathbf{5}, \quad$ 2-oxindoles 8 and 3 oxindolines 9 through base- or acid-promoted rearrangement reactions (Scheme 8). ${ }^{[13]}$ Possibly, the driving-force of these reorganizations may be related to the gain in stability associated with the $1 H$-indole, indolinone and indolone formation.

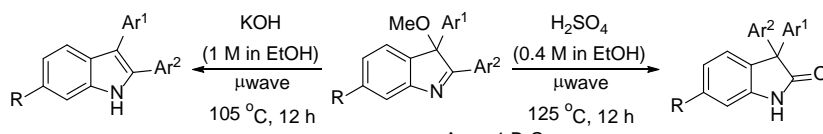

$$
\begin{aligned}
& \begin{array}{lll}
5 \mathbf{5 a b}(71 \%) & 6 \mathbf{6 a b} \mathrm{R}=\mathrm{H}, \mathrm{Ar}^{1}=\mathrm{Ar}_{2}=4-\mathrm{BrC}{ }^{1} \mathrm{H}_{4} & \mathbf{8 a b} \\
\mathbf{5 c b}(72 \%) & \mathbf{6 c b} \mathrm{C}=\mathrm{F}, \mathrm{Ar}^{1}=4-\mathrm{BrC}^{6}{ }^{6} \mathrm{H}_{2} & \mathbf{8 c b}(77 \%)
\end{array} \\
& \begin{array}{lll}
\text { 5abe }(68 \%) & \text { 6cb R }=\mathrm{F}, \mathrm{Ar}^{1}=\mathrm{Ar}_{2}=4-\mathrm{BrC}_{6} \mathrm{H}_{4}=4-\mathrm{ClC} & \mathbf{8 c b}(77 \%) \\
& \text { 6abe } \mathrm{R}=\mathrm{H}, \mathrm{Ar}^{1}=4-\mathrm{BrC}_{6} \mathrm{H}_{4}, \mathrm{Ar}^{2}=4{ }_{6} \mathrm{H}_{4} & \mathbf{8 a b e}(67 \%)
\end{array} \\
& \begin{array}{c|c}
\mu \text { wave } & \mathrm{H}_{2} \mathrm{SO}_{4} \\
95{ }^{\circ} \mathrm{C}, 12 \mathrm{~h} & (0.2 \mathrm{M} \text {. }
\end{array} \\
& 95^{\circ} \mathrm{C}, 12 \mathrm{~h} \downarrow(0.2 \mathrm{M} \text { in EtOH }) \\
& \text { (abe } \\
& \text { 9ab }(51 \%) \\
& \begin{array}{l}
\text { 9cb }(56 \%) \\
\text { 9abe }(54 \%)
\end{array}
\end{aligned}
$$

Scheme 8. Synthetic transformations of 3-methoxy-2,3diaryl-3H-indoles 6.

With thia-, selena-, and azido-(TMSethynyl)benzenes 1a-Si-SMe, 1a-Si-SeMe, and 4-Si found to be compatible with the diarylative fivemembered heterocyclization reaction, structurally different precursors were investigated to further expand the scope of the reaction to six-membered benzo-fused heterocycles. For the investigation of the scope of the sequence, a pair of potential precursors, esters $\mathbf{1 0}-\mathbf{S i}$ and sulphonamide $\mathbf{1 1}-\mathbf{S i}$, was prepared. Interestingly, isocoumarins $\mathbf{1 2}$ were obtained in fair yields by the light-driven gold/photoredox-cocatalyzed double arylation/oxycyclization of 2[(trimethylsilyl)ethynyl]benzoates 10-Si (Scheme 9). Notably, regioisomeric five-membered heterocycles were not detected, highlighting the exquisite selectivity of the sequence. Starting from 2[(trimethylsilyl)ethynyl]benzenesulfonamide 11-Si, the same diarylative protocol afforded benzosultams 13 with satisfactory vields (Scheme 10). It is apparent that in esters 10-Si and sulphonamide 11-Si the 6-endo oxy- and aza-cyclizations paths are favoured because competitive 5-exo heterocyclizations are not involved.

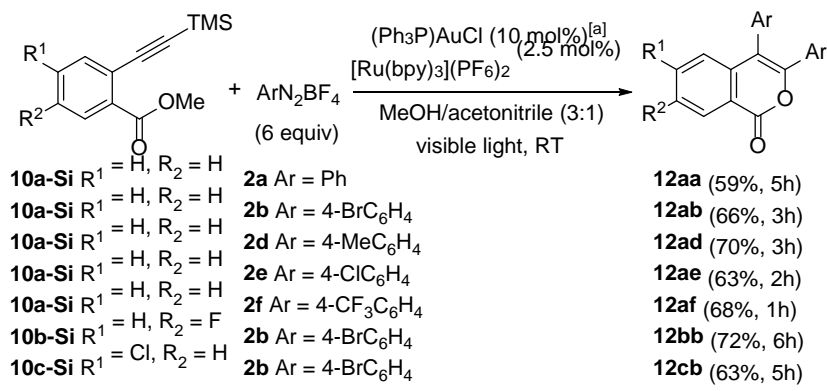

Scheme 9. Cooperative gold-photoredox catalysis for the synthesis of 3,4-diaryl- isocoumarins 12. [a] For a full conversion, the complex was added in two separated portions with a 30 min spacing.

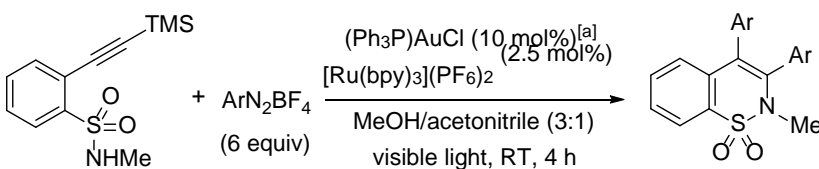

$$
\begin{aligned}
& \text { 11-Si 2b Ar }=4-\mathrm{BrC}_{6} \mathrm{H}_{4} \quad \text { 13b }(60 \%) \\
& \text { 2e } \mathrm{Ar}=4-\mathrm{ClC}_{6} \mathrm{H}_{4} \quad \text { 13e }(68 \%) \\
& 2 \mathrm{~g} \mathrm{Ar}=4-\mathrm{FC}_{6} \mathrm{H}_{4} \quad \mathbf{1 3 g}(66 \%) \\
& \text { 2i } \mathrm{Ar}=3-\mathrm{BrC}_{6} \mathrm{H}_{4} \quad 13 \mathbf{i}(58 \%)
\end{aligned}
$$

Scheme 10. Cooperative gold-photoredox catalysis for the synthesis of 3,4-diaryl-benzosultams 13.

Having in hand (trimethylsilyl)prop-2-yn-1-one 14-Si, we attempted the double arylation with arenediazonium salt $\mathbf{2 a}$ under the above reaction conditions. However, absence of reaction was observed (Scheme 11), which shows that the contiguous ketone group in the (trimethylsilyl)ethyne moiety is critical for the suppression of any HiyamaSonogashira reaction. Efforts to modified either the gold catalyst or the diazonium salt led to no improvement in reactivity. We decided to implement our planned synthesis of benzo-fused heterocycles replacing the TMS group by an aryl substituent. Convincing confirmation for the negative effect of the alkynone framework on the sila-coupling but not on the heterocyclization event, was definitively obtained by the fruitful accomplishment of the monoarylative/heterocyclization in aryl-terminated alkynone 14-Ph by which synthesis of $4 \mathrm{H}$-chromen-4ones 15 has been attained (Scheme 11). The succcesful cyclization of the heteroatom-linked alkynone core was further confirmed by the light-driven 
gold/photoredox-co-catalyzed

arylative thiacyclization reaction of the sulpha-derivative $\mathbf{1 6 - P h}$ with several diazonium salts 2 to afford $4 \mathrm{H}$ thiochromen-4-ones 17 (Scheme 12). The detected regiochemistry of both ring closures (6-endo oxy- and thia-cyclizations) is in agreement with the results of Schemes 9 and 10.

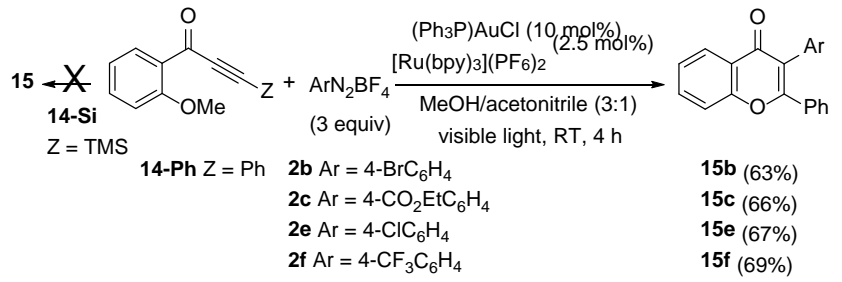

Scheme 11. Cooperative gold-photoredox catalysis for the synthesis of 3-aryl-flavones 15.

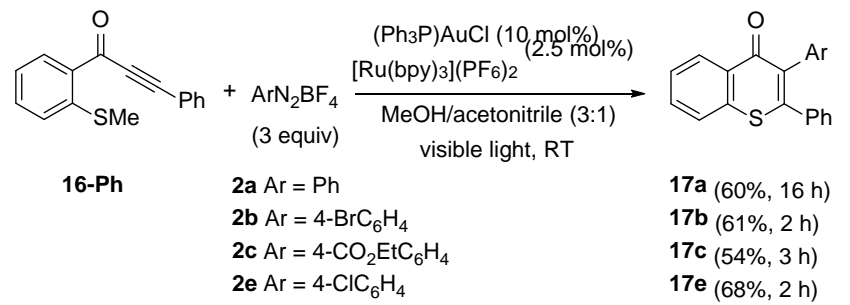

Scheme 12. Cooperative gold-photoredox catalysis for the synthesis of 3-aryl-thioflavones 17.

Despite that Hashmi ${ }^{[14]}$ and $\mathrm{Shi}^{[15]}$ have independently shown that no photoredox catalysts are required for related reactions, a control experiment verified that our reactions operates through $\left[\mathrm{Ru}(\mathrm{bpy})_{3}\right]\left(\mathrm{PF}_{6}\right)_{2}$ photoredox. Indeed, starting either from 1a-Si-SMe or $\mathbf{4 a - S i}$ no reaction occurred in the absence of the photosensitizer. The critical role of light in the domino reaction was probed when precursors 1a-Si-SMe or 4a-Si were recovered when the reactions were run with all the reagents but without irradiation. A tentative mechanistic proposal for the generation of 3-alkoxy-2,3-diaryl-3H-indoles 6 from 2[(trimethylsilyl)ethynyl]azidobenzenes $\quad \mathbf{4 - S i}$, diazonium salts $\mathbf{2}$ and light under dual goldphotoredox cocatalysis is summarized in Scheme 13. Initially, irradiation of the $\mathrm{Ru}(\mathrm{II})$-based photoredox catalyst results in the formation of an aryl radical from diazonium salts 2 after extrusion of dinitrogen (bottom catalytic cycle, right side). This key reactive species is able to be coupled with the gold(I) precatalyst to allow the formation of unstable organogold(II) intermediate 18, which rapidly evolves to the cationic organogold(III) derivative 19, a strong electrophile, through the oxidative action of $\mathrm{Ru}(\mathrm{III})$ and concomitant liberation of $\left[\mathrm{Ru}(\mathrm{bpy})_{3}\right]\left(\mathrm{PF}_{6}\right)_{2}$ into the catalytic cycle. Next, TMS-(ethynyl)azidobenzenes 4Si enter the gold catalytic sequence (bottom catalytic cycle, left side), forming complexes 4-Si-Au(III) through alkyne coordination with the gold complex. Either the transmetallation of the C(sp)-Si bond or the nucleophilic attack from the azide group can be effectively produced. Apparently, the silicon/gold interchange is preferred to the amino-auration. Then,
$\mathrm{Si}-\mathrm{Au}$ transmetallation should produce gold acetylide species 20, which suffers reductive elimination paired to aryl transfer and releases 2(arylethynyl)azidobenzenes 4-Ar and the gold(I) salt, closing the first gold catalytic cycle (bottom catalytic cycle). The formation of 3-alkoxy-2,3-diaryl-3Hindoles 6 from 2-(arylethynyl)azidobenzenes $\mathbf{4 - A r}$ requires the participation of the organogold(III) species 19 in the azacyclization event. According to the aforementioned comments, a second molecule of arenediazonium salt $\mathbf{2}$ affords the corresponding aryl radical helped by light and the photoredox catalyst (top catalytic cycle, right side). The $\mathrm{N}$ atom attack to the terminal carbon of the triple bond with respect to the azide moiety is facilitated in intermediate 4-ArAu(III) by the coordination of arylgold(III) species $\mathbf{1 9}$ with the alkyne functionality of 4-Ar. After the 5endo-dig azacyclization, the aryl transfer from the $\mathrm{Au}$ atom to the C3 indole carbon lead to intermediate $1 \mathrm{H}$ indoles 22. In concert, the gold(I) precatalyst is regenerated in this pathway (top catalytic cycle, left side). The formation of 3-alkoxy-2,3-diaryl-3Hindoles 6 requires the further attack of the alcohol with concomitant nitrogen releases.

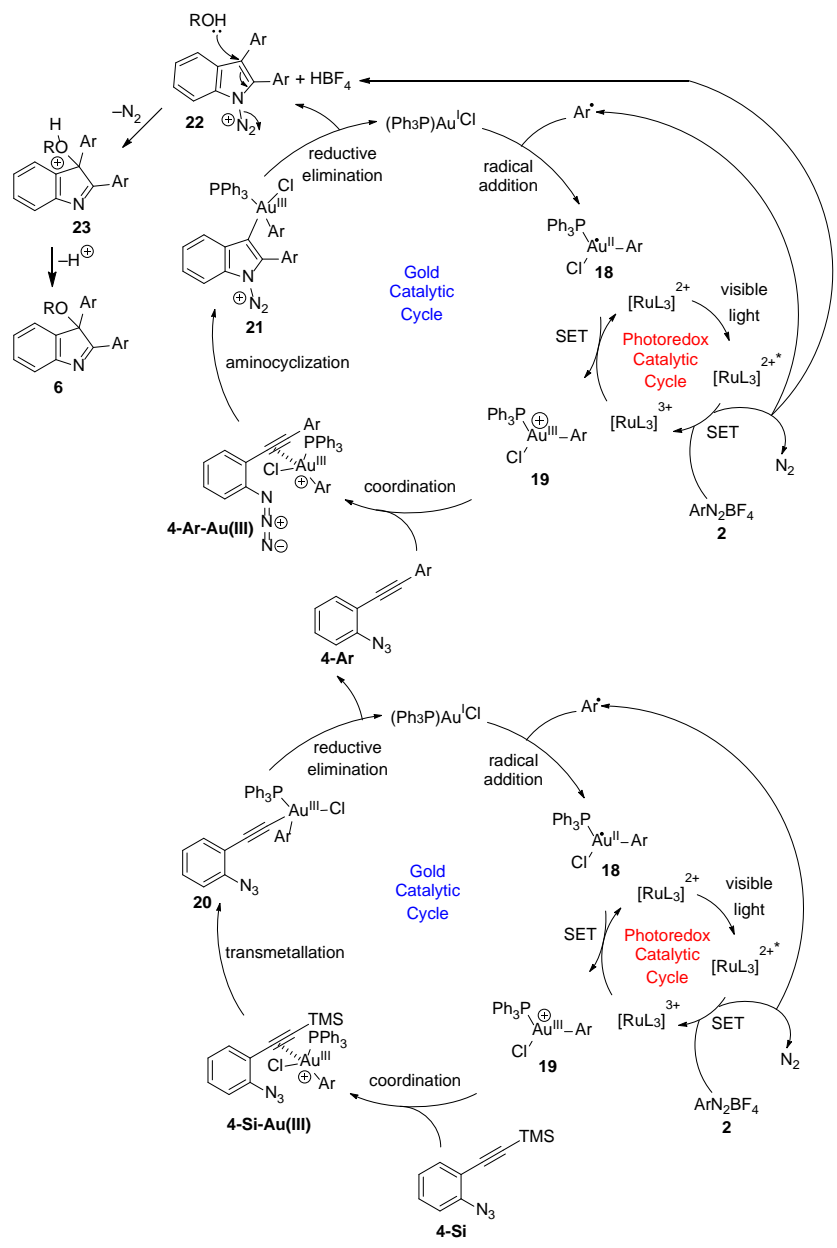

Scheme 13. Rationalization for the gold-photoredox cocatalyzed preparation of 3-alkoxy-2,3-diaryl-3H-indoles 6 from TMS-(ethynyl)azidobenzenes 4-Si and diazonium salts 2 . 


\section{Conclusions}

In conclusion, visible light-promoted and goldphotoredox catalyzed reactions of heteroatom( $\mathrm{N}, \mathrm{S}$, Se, O)-tethered alkyne derivatives with diazonium salts are totally selective to build in a controlled manner vicinal diaryl-substituted $2 H$-benzo[e][1,2]thiazine 1,1-dioxides (benzosultams), benzoselenophenes, benzothiophenes, $4 \mathrm{H}$-chromen-4-ones (flavones), $3 \mathrm{H}$ indoles, $1 H$-isochromen-1-ones (isocoumarins), and $4 H$-thiochromen-4-ones (thioflavones). Besides, the usefulness of $3 \mathrm{H}$-indoles has been probed with the facile and divergent synthesis of $1 \mathrm{H}$-indoles, 2oxindoles and 3-oxindolines.

\section{Experimental Section}

General methods: ${ }^{1} \mathrm{H}$ NMR and ${ }^{13} \mathrm{C}$ NMR spectra were recorded on a Bruker Avance AVIII-700 with cryoprobe, Bruker AMX-500, or a Bruker AMX-500, or a Bruker Avance-300 spectrometers. NMR spectra were recorded in $\mathrm{CDCl}_{3}$ solutions, except otherwise stated. Chemical shifts are given in ppm relative to TMS $\left({ }^{1} \mathrm{H}, 0.0 \mathrm{ppm}\right)$, or $\mathrm{CDCl}_{3}$ $\left({ }^{1} \mathrm{H}, 7.27 \mathrm{ppm} ;{ }^{13} \mathrm{C}, 76.9 \mathrm{ppm}\right)$, or $\mathrm{C}_{6} \mathrm{D}_{6}\left({ }^{1} \mathrm{H}, 7.16 \mathrm{ppm} ;{ }^{13} \mathrm{C}\right.$, $128.0 \mathrm{ppm})$. Low and high resolution mass spectra were taken on an AGILENT 6520 Accurate-Mass QTOF LC/MS spectrometer using the electrospray mode (ES) unless otherwise stated. IR spectra were recorded on a Bruker Tensor 27 spectrometer. All commercially available compounds were used without further purification.

General procedure for the photopromoted goldcatalyzed twofold arylation reaction of hetero-subtituted TMS-(ethynyl)benzenes 1a-Si-SMe, 1a-Si-SeMe, 4a-f-Si, 10a-c-Si, or 11-Si with diazonium salts 2. Preparation of 2,3-diaryl benzothiophenes 3-S, 2,3-diaryl benzoselenophenes 3-Se, 3-alkoxy-2,3-diaryl-3H-indoles 6, 3,4-diaryl-isocoumarins 11 , and 3,4-diarylbenzosultams 13. In a Schlenk tube in the absence of light at $-78{ }^{\circ} \mathrm{C}$ under argon atmosphere, $\mathrm{Ph}_{3} \mathrm{PAuCl}(10 \mathrm{~mol} \%)$ and $\left[\mathrm{Ru}(\mathrm{bpy})_{3]}\left(\mathrm{PF}_{6}\right)_{2}(2.5 \mathrm{~mol} \%)\right.$ were sequentially added to a solution of the corresponding arene diazonium salt $\mathbf{2}$ (6.0 equiv) in a mixture of $\mathrm{MeOH} / \mathrm{MeCN}(3: 1,5.0 \mathrm{~mL}$ ). Then, a solution of the appropriate 2[(trimethylsilyl)ethynyl]benzene 1a-Si-SMe, 1a-Si-SeMe, 4a-f-Si, 10a-c-Si, or 11-Si $(1.0 \mathrm{mmol})$ in $\mathrm{MeOH} / \mathrm{MeCN}$ (3:1, $2.5 \mathrm{~mL})$ was added dropwise and the reaction was stirred at $-78^{\circ} \mathrm{C}$ for $5 \mathrm{~min}$. For a full conversion in the case of azides $\mathbf{4}$, at the beginning the gold salt was added in two separated portions with a 30 min spacing. The reaction mixture was then warmed to room temperature and stirred under irradiation from visible light source ( $21 \mathrm{~W}$ fluorescent light bulb installed in a tool box). After disappearance of the starting material (TLC), the reaction mixture was concentrated under reduced pressure. Chromatography of the residue using hexanes/ethyl acetate or hexanes/toluene mixtures gave analytically pure compounds. The addition of $\mathrm{Et}_{3} \mathrm{~N}(2 \%)$ to the eluent was neccesary for the purification of acid sensitive $3 \mathrm{H}$-indoles $\mathbf{6}$. Spectroscopic and analytical data for pure forms of compounds 1a-Si-SMe, 1a-Si-SeMe, 4a-f-Si, 10a-c-Si, and 11-Si follow. ${ }^{[16]}$

2,3-Diaryl benzothiophene 3a-S. From $22 \mathrm{mg}(0.10 \mathrm{mmol})$ of TMS-alkyne 1a-Si-SMe, and after chromatography of the residue using hexanes as eluent, gave compound 3a-S (27 mg, 94\%) as a colorless solid; m.p. $107-109{ }^{\circ} \mathrm{C} ;{ }^{1} \mathrm{H}$ NMR $\left(300 \mathrm{MHz}, \mathrm{CDCl}_{3}, 25{ }^{\circ} \mathrm{C}\right) \delta: 7.85-7.79\left(\mathrm{~m}, 1 \mathrm{H}, \mathrm{CH}^{\mathrm{Ar}}\right)$, 7.54-7.50 (m, $\left.1 \mathrm{H}, \mathrm{CH}^{\mathrm{Ar}}\right), 7.31-7.15\left(\mathrm{~m}, 12 \mathrm{H}, 12 \mathrm{CH}^{\mathrm{Ar}}\right) ;{ }^{13} \mathrm{C}$ NMR $\left(75 \mathrm{MHz}, \mathrm{CDCl}_{3}, 25^{\circ} \mathrm{C}\right) \delta: 141.2\left(C^{\mathrm{Ar}-\mathrm{q}}\right), 139.9\left(C^{\mathrm{Ar}-}\right.$ $\left.{ }^{\mathrm{q}}\right), 139.2\left(C^{\mathrm{Ar}-\mathrm{q}}\right), 135.8\left(C^{\mathrm{Ar}-\mathrm{q}}\right), 134.6\left(C^{\mathrm{Ar}-\mathrm{q}}\right), 133.6\left(C^{\mathrm{Ar}-\mathrm{q}}\right)$, $130.8\left(2 \mathrm{CH}^{\mathrm{Ar}}\right), 130.0\left(2 \mathrm{CH}^{\mathrm{Ar}}\right), 129.0\left(2 \mathrm{CH}^{\mathrm{Ar}}\right), 128.7$ $\left(2 \mathrm{CH}^{\mathrm{Ar}}\right), 128.1\left(\mathrm{CH}^{\mathrm{Ar}}\right), 127.7\left(\mathrm{CH}^{\mathrm{Ar}}\right), 124.9\left(\mathrm{CH}^{\mathrm{Ar}}\right), 124.8$ $\left(\mathrm{CH}^{\mathrm{Ar}}\right), 123.7\left(\mathrm{CH}^{\mathrm{Ar}}\right), 122.4\left(\mathrm{CH}^{\mathrm{Ar}}\right)$; IR $\left(\mathrm{CHCl}_{3}, \mathrm{~cm}^{-1}\right)$ : $v$ 1599, 1436; HRMS (ES): calcd for $\mathrm{C}_{20} \mathrm{H}_{15} \mathrm{~S}[M+\mathrm{H}]^{+}$: 287.0889; found: 287.0898 .

2,3-Diaryl benzothiophene 3b-S. From $35 \mathrm{mg}$ ( $0.16 \mathrm{mmol})$ of TMS-alkyne 1a-Si-SMe, and after chromatography of the residue using hexanes as eluent, gave compound $\mathbf{3 b}$-S (60 mg, 86\%) as a colorless solid; m.p. $175-177{ }^{\circ} \mathrm{C} ;{ }^{1} \mathrm{H}$ NMR $\left(300 \mathrm{MHz}, \mathrm{CDCl}_{3}, 25{ }^{\circ} \mathrm{C}\right) \delta: 7.89\left(\mathrm{~m}, 1 \mathrm{H}, \mathrm{CH}^{\mathrm{Ar}}\right), 7.56(\mathrm{~m}$, $\left.3 \mathrm{H}, 3 \mathrm{CH}^{\mathrm{Ar}}\right), 7.40$ (m, 4H, 4CH $\mathrm{CH}^{\mathrm{Ar}}$, 7.19 (m, $\left.4 \mathrm{H}, 4 \mathrm{CH}^{\mathrm{Ar}}\right) ;{ }^{13} \mathrm{C}$ NMR $\left(75 \mathrm{MHz}, \mathrm{CDCl}_{3}, 25^{\circ} \mathrm{C}\right) \delta: 140.3\left(C^{\mathrm{Ar}-\mathrm{q}}\right), 138.8\left(C^{\mathrm{Ar}-}\right.$ $\left.{ }^{\mathrm{q}}\right), 138.6\left(C^{\mathrm{Ar}-\mathrm{q}}\right), 134.1\left(C^{\mathrm{Ar}-\mathrm{q}}\right), 132.9\left(C^{\mathrm{Ar}-\mathrm{q}}\right), 132.3\left(C^{\mathrm{Ar}-\mathrm{q}}\right)$, $132.1 \quad\left(2 \mathrm{CH}^{\mathrm{Ar}}\right), 132.0 \quad\left(2 \mathrm{CH}^{\mathrm{Ar}}\right), 131.7 \quad\left(2 \mathrm{CH}^{\mathrm{Ar}}\right), 131.1$ $\left(2 \mathrm{CH}^{\mathrm{Ar}}\right), 124.9\left(\mathrm{CH}^{\mathrm{Ar}}\right), 124.8\left(\mathrm{CH}^{\mathrm{Ar}}\right), 123.1\left(\mathrm{CH}^{\mathrm{Ar}}\right), 122.3$ $\left(C^{\mathrm{Ar}-\mathrm{q}}\right), 122.2\left(\mathrm{CH}^{\mathrm{Ar}}\right), 121.8\left(C^{\mathrm{Ar}-\mathrm{q}}\right)$; IR $\left(\mathrm{CHCl}_{3}, \mathrm{~cm}^{-1}\right)$ : $v$ 1533, 1484; HRMS (ES): calcd for $\mathrm{C}_{20} \mathrm{H}_{13} \mathrm{Br}_{2} \mathrm{~S}[\mathrm{M}+\mathrm{H}]^{+}$: 442.9099; found: 442.9087.

2,3-Diaryl benzoselenophene 3d-Se. From 26 mg $(0.10$ mmol) of TMS-alkyne 1a-Si-SeMe, and after chromatography of the residue using hexanes as eluent, gave compound 3d-Se (28 mg, 60\%) as a colorless solid; m.p. $140-142{ }^{\circ} \mathrm{C} ;{ }^{1} \mathrm{H}$ NMR $\left(300 \mathrm{MHz}, \mathrm{CDCl}_{3}, 25^{\circ} \mathrm{C}\right) \delta: 8.08-$ $8.04\left(\mathrm{~m}, 1 \mathrm{H}, \mathrm{CH}^{\mathrm{Ar}}\right), 7.67-7.63\left(\mathrm{~m}, 1 \mathrm{H}, \mathrm{CH}^{\mathrm{Ar}}\right), 7.47-7.30(\mathrm{~m}$, $\left.8 \mathrm{H}, 8 \mathrm{CH}^{\mathrm{Ar}}\right), 7.21-7.17\left(\mathrm{~m}, 2 \mathrm{H}, 2 \mathrm{CH}^{\mathrm{Ar}}\right), 2.56\left(\mathrm{~s}, \mathrm{CH}_{3}\right), 2.46$ (s, $\left.\mathrm{CH}_{3}\right) ;{ }^{13} \mathrm{C}$ NMR $\left(75 \mathrm{MHz}, \mathrm{CDCl}_{3}, 25^{\circ} \mathrm{C}\right) \delta: 143.8\left(\mathrm{C}^{\mathrm{Ar}-}\right.$ $\left.{ }^{\mathrm{q}}\right), 143.2\left(C^{\mathrm{Ar}-\mathrm{q}}\right), 140.2\left(C^{\mathrm{Ar}-\mathrm{q}}\right), 137.4\left(C^{\mathrm{Ar}-\mathrm{q}}\right), 136.9\left(C^{\mathrm{Ar}-\mathrm{q}}\right)$, $135.9\left(C^{\mathrm{Ar}-\mathrm{q}}\right), 133.8\left(C^{\mathrm{Ar}-\mathrm{q}}\right), 133.4\left(C^{\mathrm{Ar}-\mathrm{q}}\right), 130.4\left(2 \mathrm{CH}^{\mathrm{Ar}}\right)$, $129.7\left(2 \mathrm{CH}^{\mathrm{Ar}}\right), 129.4\left(2 \mathrm{CH}^{\mathrm{Ar}}\right), 129.1\left(2 \mathrm{CH}^{\mathrm{Ar}}\right), 125.6\left(\mathrm{CH}^{\mathrm{Ar}}\right)$, 125.2 $\left(\mathrm{CH}^{\mathrm{Ar}}\right), 124.6\left(2 \mathrm{CH}^{\mathrm{Ar}}\right) ;{ }^{77} \mathrm{Se} \mathrm{NMR}\left(95 \mathrm{MHz}, \mathrm{CDCl}_{3}\right.$, $\left.25{ }^{\circ} \mathrm{C}\right) \delta$ : 528.9 (s, 1Se); IR $\left(\mathrm{CHCl}_{3}, \mathrm{~cm}^{-1}\right): v$ 1506, 1439, 810; HRMS (ES): calcd for $\mathrm{C}_{22} \mathrm{H}_{19} \mathrm{Se}[M+\mathrm{H}]^{+}$: 363.0647; found: 363.0632 .

2,3-Diaryl benzoselenophene 3e-Se. From 26 mg (0.10 mmol) of TMS-alkyne 1a-Si-SeMe, and after chromatography of the residue using hexanes as eluent, gave compound 3e-Se (21 mg, 52\%) as a colorless solid; m.p. 198-200 ${ }^{\circ} \mathrm{C} ;{ }^{1} \mathrm{H}$ NMR $\left(300 \mathrm{MHz}, \mathrm{CDCl}_{3}, 25^{\circ} \mathrm{C}\right) \delta: 7.97-$ $7.94\left(\mathrm{~m}, 1 \mathrm{H}, \mathrm{CH}^{\mathrm{Ar}}\right), 7.52-7.49\left(\mathrm{~m}, 1 \mathrm{H}, \mathrm{CH}^{\mathrm{Ar}}\right), 7.42-7.27(\mathrm{~m}$, $\left.4 \mathrm{H}, 4 \mathrm{CH}^{\mathrm{Ar}}\right)$, 7.26-7.17 (m, 6H, 6CH $\left.\mathrm{CH}^{\mathrm{Ar}}\right) ;{ }^{13} \mathrm{C}$ NMR $(75 \mathrm{MHz}$, $\left.\mathrm{CDCl}_{3}, 25^{\circ} \mathrm{C}\right) \delta: 143.0\left(C^{\mathrm{Ar}-\mathrm{q}}\right), 142.3\left(C^{\mathrm{Ar}-\mathrm{q}}\right), 140.5\left(C^{\mathrm{Ar}-\mathrm{q}}\right)$, $135.6\left(C^{\mathrm{Ar}-\mathrm{q}}\right), 134.9\left(C^{\mathrm{Ar}-\mathrm{q}}\right), 134.4\left(C^{\mathrm{Ar}-\mathrm{q}}\right), 133.8\left(C^{\mathrm{Ar}-\mathrm{q}}\right)$, $133.6\left(C^{\mathrm{Ar}-\mathrm{q}}\right), 131.9\left(2 \mathrm{CH}^{\mathrm{Ar}}\right), 131.0\left(2 \mathrm{CH}^{\mathrm{Ar}}\right), 129.1\left(2 \mathrm{CH}^{\mathrm{Ar}}\right)$, $128.7\left(2 \mathrm{CH}^{\mathrm{Ar}}\right), 125.5\left(\mathrm{CH}^{\mathrm{Ar}}\right), 125.4\left(\mathrm{CH}^{\mathrm{Ar}}\right), 125.2\left(\mathrm{CH}^{\mathrm{Ar}}\right)$, $125.0\left(\mathrm{CH}^{\mathrm{Ar}}\right) ;{ }^{77} \mathrm{Se} \mathrm{NMR}\left(95 \mathrm{MHz}, \mathrm{CDCl}_{3}, 25^{\circ} \mathrm{C}\right) \delta: 538.9$ (s, 1Se); IR $\left(\mathrm{CHCl}_{3}, \mathrm{~cm}^{-1}\right): v 1484,1089,833$; HRMS (ES): calcd for $\mathrm{C}_{20} \mathrm{H}_{13} \mathrm{Cl}_{2} \mathrm{Se}[M+\mathrm{H}]^{+}$: 402.9554; found: 402.9570. 
3-Alkoxy-2,3-diaryl-3H-indole 6ab. From $30 \mathrm{mg}(0.14$ mmol) of TMS-azide 4a-Si, and after chromatography of the residue using hexanes/Et ${ }_{2} \mathrm{O}(97: 3)$ containing $\mathrm{NEt}_{3}(2 \%)$ as eluent, gave compound $\mathbf{6 a b}$ (40 mg, 63\%) as a yellow oil; ${ }^{1} \mathrm{H}$ NMR (500 MHz, $\left.\mathrm{CDCl}_{3}, 25^{\circ} \mathrm{C}\right) \delta: 7.97\left(\mathrm{~m}, 2 \mathrm{H}, 2 \mathrm{CH}^{\mathrm{Ar}}\right.$ ), 7.68 (d, $1 \mathrm{H}, J=7.7 \mathrm{~Hz}, \mathrm{CH}^{\mathrm{Ar}}$ ), 7.51 (m, 2H, 2CH $\left.{ }^{\mathrm{Ar}}\right), 7.41$ (m, 1H, CH ${ }^{\mathrm{Ar}}$ ), $7.37\left(\mathrm{~m}, 2 \mathrm{H}, 2 \mathrm{CH}^{\mathrm{Ar}}\right), 7.22(\mathrm{~d}, 1 \mathrm{H}, J=6.6$ $\mathrm{Hz}, \mathrm{CH}^{\mathrm{Ar}}$ ), 7.19 (m, 2H, 2CH $\left.\mathrm{CH}^{\mathrm{Ar}}\right), 7.11$ (d, $1 \mathrm{H}, J=6.9 \mathrm{~Hz}$, $\mathrm{CH}^{\mathrm{Ar}}$ ), 3.06 (s, $\left.3 \mathrm{H}, \mathrm{OCH}_{3}\right) ;{ }^{13} \mathrm{C}$ NMR (125 $\mathrm{MHz} \mathrm{CDCl}_{3}$, $\left.25^{\circ} \mathrm{C}\right) \delta: 177.2(\mathrm{~N}=\mathrm{C}), 153.2\left(C^{\mathrm{Ar}-\mathrm{q}}\right), 139.7\left(C^{\mathrm{Ar}-\mathrm{q}}\right), 138.3$ $\left(C^{\mathrm{Ar}-\mathrm{q}}\right), 131.9\left(2 \mathrm{CH}^{\mathrm{Ar}}\right), 130.2\left(\mathrm{CH}^{\mathrm{Ar}}\right), 130.0\left(C^{\mathrm{Ar}-\mathrm{q}}\right), 129.9$ $\left(2 \mathrm{CH}^{\mathrm{Ar}}\right), 127.2\left(\mathrm{CH}^{\mathrm{Ar}}\right), 126.4\left(C^{\mathrm{Ar}-\mathrm{q}}\right), 126.2\left(2 \mathrm{CH}^{\mathrm{Ar}}\right), 123.4$ $\left(\mathrm{CH}^{\mathrm{Ar}}\right), 121.7\left(C^{\mathrm{Ar}-\mathrm{q}}\right), 121.6\left(\mathrm{CH}^{\mathrm{Ar}}\right), 92.9\left(\mathrm{OC}^{\mathrm{q}}\right), 52.6$ $\left(\mathrm{OCH}_{3}\right)$; IR $\left(\mathrm{CHCl}_{3}, \mathrm{~cm}^{-1}\right): v 1728(\mathrm{~N}=\mathrm{C}), 1079(\mathrm{C}-\mathrm{O})$; HRMS (ES): calcd for $\mathrm{C}_{21} \mathrm{H}_{16} \mathrm{Br}_{2} \mathrm{NO}[M+\mathrm{H}]^{+}$: 455.9593; found: 455.9601.

3-Alkoxy-2,3-diaryl-3H-indole $\mathbf{6 a b}-\mathbf{C D}_{3}$. The reaction was runned in $\mathrm{CD}_{3} \mathrm{OD}$ instead $\mathrm{MeOH}$. From $30 \mathrm{mg}(0.14$ $\mathrm{mmol}$ ) of azide $\mathbf{4 a - S i}$, and after chromatography of the residue using hexanes/ $\mathrm{Et}_{2} \mathrm{O}(97: 3)$ as eluent containing $\mathrm{NEt}_{3}$ (2\%), gave compound $\mathbf{6 a b}-\mathbf{C D}_{3}$ (38 mg, 59\%) as a yellow oil; ${ }^{1} \mathrm{H}$ NMR (300 MHz, $\left.\mathrm{CDCl}_{3}, 25{ }^{\circ} \mathrm{C}\right) \delta: 7.87(\mathrm{~m}$, $2 \mathrm{H}, 2 \mathrm{CH}^{\mathrm{Ar}}$ ), 7.58 (d, $\left.1 \mathrm{H}, J=7.7 \mathrm{~Hz}, \mathrm{CH}^{\mathrm{Ar}}\right), 7.41(\mathrm{~m}, 2 \mathrm{H}$, $2 \mathrm{CH}^{\mathrm{Ar}}$ ), $7.29\left(\mathrm{~m}, 3 \mathrm{H}, 3 \mathrm{CH}^{\mathrm{Ar}}\right), 7.10\left(\mathrm{~m}, 2 \mathrm{H}, 2 \mathrm{CH}^{\mathrm{Ar}}\right), 7.01$ (d, $\left.1 \mathrm{H}, J=6.8 \mathrm{~Hz}, \mathrm{CH}^{\mathrm{Ar}}\right) ;{ }^{13} \mathrm{C} \mathrm{NMR}\left(75 \mathrm{MHz}, \mathrm{CDCl}_{3}, 25^{\circ} \mathrm{C}\right)$ $\delta: 177.2(\mathrm{~N}=\mathrm{C}), 153.3\left(C^{\mathrm{Ar}-\mathrm{q}}\right), 139.7\left(C^{\mathrm{Ar}-\mathrm{q}}\right), 138.3\left(C^{\mathrm{Ar}-\mathrm{q}}\right)$, $131.9\left(2 \mathrm{CH}^{\mathrm{Ar}}\right), 130.2\left(\mathrm{CH}^{\mathrm{Ar}}\right), 130.1\left(C^{\mathrm{Ar}-\mathrm{q}}\right), 129.9\left(2 \mathrm{CH}^{\mathrm{Ar}}\right)$, $127.2\left(\mathrm{CH}^{\mathrm{Ar}}\right), 126.4\left(C^{\mathrm{Ar}-\mathrm{q}}\right), 126.2\left(2 \mathrm{CH}^{\mathrm{Ar}}\right), 123.4\left(\mathrm{CH}^{\mathrm{Ar}}\right)$, $121.7\left(C^{\mathrm{Ar}-\mathrm{q}}\right), 121.6\left(\mathrm{CH}^{\mathrm{Ar}}\right), 92.9\left(\mathrm{OC}^{\mathrm{q}}\right) ; \mathrm{D}\left({ }^{2} \mathrm{H}\right)$ NMR $(107$ $\left.\mathrm{MHz}, \mathrm{CDCl}_{3}, 25^{\circ} \mathrm{C}\right) \delta$ : 3.04; IR $\left(\mathrm{CHCl}_{3}, \mathrm{~cm}^{-1}\right): v 1723$ $(\mathrm{N}=\mathrm{C}), \quad 1076$ (C-O); HRMS (ES): calcd for $\mathrm{C}_{21} \mathrm{H}_{13} \mathrm{D}_{3} \mathrm{Br}_{2} \mathrm{NO}[M+\mathrm{H}]^{+}$: 458.9782; found: 458.9784 .

3,4-Diaryl-isocoumarin 12ad. From $40 \mathrm{mg}$ (0.18 mmol) of TMS-alkyne 10a-Si, and after chromatography of the residue using hexanes/ethyl acetate (95:5) as eluent, gave compound 12ad (41 mg, 70\%) as a colorless solid; m.p. 165-167 ${ }^{\circ} \mathrm{C}$; ${ }^{1} \mathrm{H}$ NMR $\left(300 \mathrm{MHz}, \mathrm{CDCl}_{3}, 25{ }^{\circ} \mathrm{C}\right) \delta: 8.39$ (d, $\left.1 \mathrm{H}, J=7.8 \mathrm{~Hz}, \mathrm{CH}^{\mathrm{Ar}}\right), 7.62\left(\mathrm{~m}, 1 \mathrm{H}, \mathrm{CH}^{\mathrm{Ar}}\right), 7.50(\mathrm{t}, 1 \mathrm{H}$, $\mathrm{CH}^{\mathrm{Ar}}$ ), 7.19 (m, 7H, 7CH ${ }^{\mathrm{Ar}}$ ), 7.01 (m, 2H, 2CH $\left.{ }^{\mathrm{Ar}}\right), 2.43$ (s, $\left.3 \mathrm{H}, \mathrm{CH}_{3}\right), 2.30\left(\mathrm{~s}, 3 \mathrm{H}, \mathrm{CH}_{3}\right) ;{ }^{13} \mathrm{C} \mathrm{NMR} \mathrm{(75} \mathrm{MHz}, \mathrm{CDCl}_{3}$, $\left.25^{\circ} \mathrm{C}\right) \delta: 162.4(\mathrm{C}=\mathrm{O}), 150.9(C=\mathrm{C}), 139.2\left(C^{\mathrm{Ar}-\mathrm{q}}\right), 139.0$ $\left(C^{\mathrm{Ar}-\mathrm{q}}\right), 137.8\left(C^{\mathrm{Ar}-\mathrm{q}}\right), 134.5\left(\mathrm{CH}^{\mathrm{Ar}}\right), 131.4\left(C^{\mathrm{Ar}-\mathrm{q}}\right), 131.0$ $\left(2 \mathrm{CH}^{\mathrm{Ar}}\right), 130.1\left(C^{\mathrm{Ar}-\mathrm{q}}\right), 129.8\left(2 \mathrm{CH}^{\mathrm{Ar}}\right), 129.4\left(\mathrm{CH}^{\mathrm{Ar}}\right), 129.0$ $\left(2 \mathrm{CH}^{\mathrm{Ar}}\right), 128.6\left(2 \mathrm{CH}^{\mathrm{Ar}}\right), 127.8\left(\mathrm{CH}^{\mathrm{Ar}}\right), 125.3\left(\mathrm{CH}^{\mathrm{Ar}}\right), 120.3$ $\left(C^{\mathrm{Ar}-\mathrm{q}}\right), 116.3(\mathrm{C}=\mathrm{C}), 21.3\left(\mathrm{CH}_{3}\right), 21.2\left(\mathrm{CH}_{3}\right)$; IR $\left(\mathrm{CHCl}_{3}\right.$, $\left.\mathrm{cm}^{-1}\right)$ : $v 1715(\mathrm{C}=\mathrm{O})$; HRMS (ES): calcd for $\mathrm{C}_{23} \mathrm{H}_{19} \mathrm{O}_{2}[M$ $+\mathrm{H}]^{+}$: 327.1380; found: 327.1388.

3,4-Diaryl-isocoumarin 12af. From $35 \mathrm{mg}$ ( $0.15 \mathrm{mmol})$ of TMS-alkyne 10a-Si, and after chromatography of the residue using hexanes/ethyl acetate (95:5) as eluent, gave compound 12af (44 mg, 68\%) as a colorless solid; m.p. 155$157^{\circ} \mathrm{C} ;{ }^{1} \mathrm{H}$ NMR (300 MHz, $\mathrm{CDCl}_{3}, 25^{\circ} \mathrm{C}$ ) $\delta: 8.45$ (dd, $1 \mathrm{H}$, $\left.J=7.9,1.3 \mathrm{~Hz}, \mathrm{CH}^{\mathrm{Ar}}\right), 7.71\left(\mathrm{~m}, 3 \mathrm{H}, 3 \mathrm{CH}^{\mathrm{Ar}}\right), 7.60(\mathrm{~m}, 1 \mathrm{H}$, $\left.\mathrm{CH}^{\mathrm{Ar}}\right), 7.50\left(\mathrm{~m}, 2 \mathrm{H}, 2 \mathrm{CH}^{\mathrm{Ar}}\right), 7.43\left(\mathrm{~m}, 4 \mathrm{H}, 4 \mathrm{CH}^{\mathrm{Ar}}\right), 7.13(\mathrm{~d}$, $\left.1 \mathrm{H}, J=7.8 \mathrm{~Hz}, \mathrm{CH}^{\mathrm{Ar}}\right) ;{ }^{13} \mathrm{C}$ NMR $\left(75 \mathrm{MHz}, \mathrm{CDCl}_{3}, 25^{\circ} \mathrm{C}\right)$ $\delta: 161.4(\mathrm{C}=\mathrm{O}), 149.7(C=\mathrm{C}), 137.7\left(\mathrm{~d}, J_{C F}=1.3 \mathrm{~Hz}, C^{\mathrm{Ar}-\mathrm{q}}\right)$, $137.6\left(C^{\mathrm{Ar}-\mathrm{q}}\right), 135.9\left(\mathrm{~d}, J_{C F}=1.0 \mathrm{~Hz}, C^{\mathrm{Ar}-\mathrm{q}}\right), 135.0\left(\mathrm{CH}^{\mathrm{Ar}}\right)$, $131.7\left(2 \mathrm{CH}^{\mathrm{Ar}}\right), 131.0\left(\mathrm{q}, J_{C F}=32.7 \mathrm{~Hz}, C^{\mathrm{Ar}-\mathrm{q}}-\mathrm{CF}_{3}\right), 130.8$ (q, $\left.J_{C F}=32.8 \mathrm{~Hz}, C^{\mathrm{Ar}-\mathrm{q}}-\mathrm{CF}_{3}\right), 129.9\left(\mathrm{CH}^{\mathrm{Ar}}\right), 129.5\left(2 \mathrm{CH}^{\mathrm{Ar}}\right)$,
$129.0\left(\mathrm{CH}^{\mathrm{Ar}}\right), 126.3\left(\mathrm{q}, J_{C F}=3.7 \mathrm{~Hz}, 2 \mathrm{CH}^{\mathrm{Ar}}\right), 125.2\left(\mathrm{CH}^{\mathrm{Ar}}\right)$, 125.1 (q, $J=3.8 \mathrm{~Hz}, 2 \mathrm{CH}^{\mathrm{Ar}}$ ), $123.8\left(\mathrm{q}, J_{C F}=272.4 \mathrm{~Hz}, \mathrm{CF}_{3}\right.$ ), $123.6\left(\mathrm{q}, J_{C F}=272.4 \mathrm{~Hz}, \mathrm{CF}_{3}\right), 120.5\left(C^{\mathrm{Ar}-\mathrm{q}}\right), 116.90(\mathrm{C}=C)$, $103.4\left(\mathrm{CH}^{\mathrm{Ar}}\right) ;{ }^{19} \mathrm{~F}$ NMR $\left(282 \mathrm{MHz}, \mathrm{CDCl}_{3}, 25^{\circ} \mathrm{C}\right):-62.9(\mathrm{~s}$, $\left.3 \mathrm{~F}, \mathrm{CF}_{3}\right),-63.2$ (s, 3F, $\left.\mathrm{CF}_{3}\right)$; IR $\left(\mathrm{CHCl}_{3}, \mathrm{~cm}^{-1}\right): v 1735$ $(\mathrm{C}=\mathrm{O})$; HRMS (ES): calcd for $\mathrm{C}_{23} \mathrm{H}_{13} \mathrm{~F}_{6} \mathrm{O}_{2}[M+\mathrm{H}]^{+}$: 435.0814; found: 435.0814 .

3,4-Diaryl-isocoumarin 12bb. From $41 \mathrm{mg}$ ( $0.11 \mathrm{mmol})$ of TMS-alkyne 10b-Si, and after chromatography of the residue using hexanes/ethyl acetate (95:5) as eluent, gave compound 12bb (37 mg, 72\%) as a colorless solid; m.p. $142-144{ }^{\circ} \mathrm{C} ;{ }^{1} \mathrm{H}$ NMR $\left(300 \mathrm{MHz}, \mathrm{CDCl}_{3}, 25^{\circ} \mathrm{C}\right) \delta: 8.06$ (dd, $\left.1 \mathrm{H}, J=8.3,2.8 \mathrm{~Hz}, \mathrm{CH}^{\mathrm{Ar}}\right), 7.60\left(\mathrm{~m}, 2 \mathrm{H}, 2 \mathrm{CH}^{\mathrm{Ar}}\right), 7.39(\mathrm{~m}$, $\left.3 \mathrm{H}, 3 \mathrm{CH}^{\mathrm{Ar}}\right), 7.16\left(\mathrm{~m}, 5 \mathrm{H}, 5 \mathrm{CH}^{\mathrm{Ar}}\right) ;{ }^{13} \mathrm{C} \mathrm{NMR}(75 \mathrm{MHz}$, $\mathrm{CDCl}_{3}, 25^{\circ} \mathrm{C}$ ) $\delta: 162.0$ (d, $\left.J_{C F}=251.5 \mathrm{~Hz}, C^{\mathrm{Ar}-\mathrm{q}}-\mathrm{F}\right), 163.7$ (d, $\left.J_{C F}=3.5 \mathrm{~Hz}, \mathrm{C}=\mathrm{O}\right), 149.5$ (d, $J_{C F}=2.6 \mathrm{~Hz}, C=\mathrm{C}$ ), 134.7 $\left(\mathrm{d}, J_{C F}=2.7 \mathrm{~Hz}, C^{\mathrm{Ar}-\mathrm{q}}\right), 132.7\left(2 \mathrm{CH}^{\mathrm{Ar}}\right), 132.6\left(2 \mathrm{CH}^{\mathrm{Ar}}\right), 131.4$ $\left(2 \mathrm{CH}^{\mathrm{Ar}}\right), 131.2\left(C^{\mathrm{Ar}-\mathrm{q}}\right), 130.6\left(2 \mathrm{CH}^{\mathrm{Ar}}\right), 127.7\left(\mathrm{~d}, J_{C F}=7.8\right.$ $\left.\mathrm{Hz}, \mathrm{CH}^{\mathrm{Ar}}\right), 123.8\left(C^{\mathrm{Ar}-\mathrm{q}}\right), 123.3\left(C^{\mathrm{Ar}-\mathrm{q}}\right), 123.1\left(\mathrm{~d}, J_{C F}=22.8\right.$ $\left.\mathrm{Hz}, \mathrm{CH}^{\mathrm{Ar}}\right), 122.9\left(C^{\mathrm{Ar}-\mathrm{q}}\right), 122.2\left(\mathrm{~d}, J_{C F}=8.2 \mathrm{~Hz}, C^{\mathrm{Ar}-\mathrm{q}}\right)$, $115.5\left(\mathrm{~d}, J_{C F}=0.9 \mathrm{~Hz}, \mathrm{C}=C\right), 115.3\left(\mathrm{~d}, J_{C F}=23.3 \mathrm{~Hz}\right.$, $\mathrm{CH}^{\mathrm{Ar}}$ ); ${ }^{19} \mathrm{~F}$ NMR $\left(282 \mathrm{MHz}, \mathrm{CDCl}_{3}, 25^{\circ} \mathrm{C}\right):-110.31$ (s, $1 \mathrm{~F}$, $\mathrm{C}-\mathrm{F})$; IR $\left(\mathrm{CHCl}_{3}, \mathrm{~cm}^{-1}\right): v 1737(\mathrm{C}=\mathrm{O})$; HRMS (ES): calcd for $\mathrm{C}_{21} \mathrm{H}_{11} \mathrm{Br}_{2} \mathrm{FO}_{2}[M+\mathrm{H}]^{+}$: 472.9183; found: 472.9192 .

3,4-Diaryl-benzosultam 13e. From $26 \mathrm{mg}$ (0.10 mmol) of TMS-alkyne 11-Si, and after chromatography of the residue using hexanes/ethyl acetate (8:2) as eluent, gave compound 13e (28 mg, 68\%) as a colorless oil; ${ }^{1} \mathrm{H}$ NMR (300 MHz, $\left.\mathrm{CDCl}_{3}, 25^{\circ} \mathrm{C}\right) \delta: 8.00-7.96\left(\mathrm{~m}, 1 \mathrm{H}, \mathrm{CH}^{\mathrm{Ar}}\right), 7.55-7.53(\mathrm{~m}$, $2 \mathrm{H}, 2 \mathrm{CH}^{\mathrm{Ar}}$ ), 7.30-7.28 (m, 3H, 3CH $\left.{ }^{\mathrm{Ar}}\right), 7.24-7.22(\mathrm{~m}, 4 \mathrm{H}$, $\left.2 \mathrm{CH}^{\mathrm{Ar}}\right), 7.15\left(\mathrm{~d}, 2 \mathrm{H}, \mathrm{J}=7.9 \mathrm{~Hz}, 2 \mathrm{CH}^{\mathrm{Ar}}\right), 2.99\left(\mathrm{~s}, \mathrm{CH}_{3}\right) ;{ }^{13} \mathrm{C}$ NMR (75 MHz, $\left.\mathrm{CDCl}_{3}, 25^{\circ} \mathrm{C}\right) \delta: 140.0\left(C^{\mathrm{Ar}-\mathrm{q}}\right), 134.9\left(C^{\mathrm{Ar}-}\right.$ $\left.{ }^{\mathrm{q}}\right), 134.1\left(C^{\mathrm{Ar}-\mathrm{q}}\right), 133.6\left(C^{\mathrm{Ar}-\mathrm{q}}\right), 133.4\left(C^{\mathrm{Ar}-\mathrm{q}}\right), 132.5\left(2 \mathrm{CH}^{\mathrm{Ar}}\right)$, $132.3\left(C^{\mathrm{Ar}-\mathrm{q}}\right), 131.8\left(\mathrm{CH}^{\mathrm{Ar}}\right), 131.6\left(C^{\mathrm{Ar}-\mathrm{q}}\right), 131.3\left(2 \mathrm{CH}^{\mathrm{Ar}}\right)$, $128.6\left(2 \mathrm{CH}^{\mathrm{Ar}}\right), 128.5\left(2 \mathrm{CH}^{\mathrm{Ar}}\right), 128.2\left(\mathrm{CH}^{\mathrm{Ar}}\right), 127.0\left(\mathrm{CH}^{\mathrm{Ar}}\right)$, $123.4\left(C^{\mathrm{Ar}-\mathrm{q}}\right), 122.0\left(\mathrm{CH}^{\mathrm{Ar}}\right), 34.5\left(\mathrm{CH}_{3}\right)$; IR $\left(\mathrm{CHCl}_{3}, \mathrm{~cm}^{-1}\right)$ : $v 1588(\mathrm{C}=\mathrm{C}), 1485,1337(\mathrm{O}=\mathrm{S}=\mathrm{O})$; HRMS (ES): calcd for $\mathrm{C}_{21} \mathrm{H}_{16} \mathrm{Cl}_{2} \mathrm{NO}_{2} \mathrm{~S}[M+\mathrm{H}]^{+}$: 416.0273; found: 416.0277 .

3,4-Diaryl-benzosultam 13g. From $26 \mathrm{mg}$ (0.10 mmol) of TMS-alkyne 11-Si, and after chromatography of the residue using hexanes/ethyl acetate (8:2) as eluent, gave compound $13 g$ (25 mg, 66\%) as a colorless oil; ${ }^{1} \mathrm{H}$ NMR (300 MHz, $\left.\mathrm{CDCl}_{3}, 25^{\circ} \mathrm{C}\right) \delta: 7.92-7.89\left(\mathrm{~m}, 1 \mathrm{H}, \mathrm{CH}^{\mathrm{Ar}}\right), 7.48-7.46(\mathrm{~m}$, $\left.2 \mathrm{H}, 2 \mathrm{CH}^{\mathrm{Ar}}\right), 7.31-7.25\left(\mathrm{~m}, 3 \mathrm{H}, 3 \mathrm{CH}^{\mathrm{Ar}}\right), 7.20-7.05(\mathrm{~m}, 2 \mathrm{H}$, $\left.2 \mathrm{CH}^{\mathrm{Ar}}\right), 7.02-6.91\left(\mathrm{~m}, 4 \mathrm{H}, 4 \mathrm{CH}^{\mathrm{Ar}}\right), 2.94\left(\mathrm{~s}, \mathrm{CH}_{3}\right) ;{ }^{13} \mathrm{C} \mathrm{NMR}$ $\left(75 \mathrm{MHz}, \mathrm{CDCl}_{3}, 25^{\circ} \mathrm{C}\right) \delta: 162.5\left(\mathrm{~d}, 1 \mathrm{H}, J_{\mathrm{CF}}=250.0 \mathrm{~Hz}\right.$, $\left.C^{\mathrm{Ar}-\mathrm{q}}-\mathrm{F}\right), 162.0\left(\mathrm{~d}, 1 \mathrm{H}, J_{\mathrm{CF}}=250.0 \mathrm{~Hz}, C^{\mathrm{Ar}-\mathrm{q}}-\mathrm{F}\right), 140.3\left(C^{\mathrm{Ar}-}\right.$ q), $133.7\left(C^{\mathrm{Ar}-\mathrm{q}}\right), 132.9162 .0\left(\mathrm{~d}, 2 \mathrm{H}, J_{\mathrm{CF}}=8.3 \mathrm{~Hz}, 2 \mathrm{CH}^{\mathrm{Ar}}\right)$, $131.9\left(\mathrm{~d}, 2 \mathrm{H}, J_{\mathrm{CF}}=8.3 \mathrm{~Hz}, 2 \mathrm{CH}^{\mathrm{Ar}}\right), 131.8\left(\mathrm{CH}^{\mathrm{Ar}}\right), 131.6$ $\left(C^{\mathrm{Ar}-\mathrm{q}}\right), 131.5\left(C^{\mathrm{Ar}-\mathrm{q}}\right), 130.0\left(C^{\mathrm{Ar}-\mathrm{q}}\right), 128.0\left(\mathrm{CH}^{\mathrm{Ar}}\right), 127.0$ $\left(\mathrm{CH}^{\mathrm{Ar}}\right), 123.4\left(C^{\mathrm{Ar}-\mathrm{q}}\right), 121.9\left(\mathrm{CH}^{\mathrm{Ar}}\right), 115.2\left(\mathrm{~d}, 4 \mathrm{H}, J_{\mathrm{CF}}=22.3\right.$ $\left.\mathrm{Hz}, 4 \mathrm{CH}^{\mathrm{Ar}}\right), 34.4\left(\mathrm{~s}, \mathrm{CH}_{3}\right) ;{ }^{19} \mathrm{~F} \mathrm{NMR}\left(282 \mathrm{MHz} \mathrm{CDCl}_{3}, 25\right.$ $\left.{ }^{\circ} \mathrm{C}\right):-111.4$ (s, 1F, C-F), -114.3 (s, 1F, C-F); IR $\left(\mathrm{CHCl}_{3}\right.$, $\left.\mathrm{cm}^{-1}\right)$ : $v 1585(\mathrm{C}=\mathrm{C}), 1487,1335(\mathrm{O}=\mathrm{S}=\mathrm{O})$; HRMS (ES): calcd for $\mathrm{C}_{21} \mathrm{H}_{16} \mathrm{~F}_{2} \mathrm{NO}_{2} \mathrm{~S}[M+\mathrm{H}]^{+}$: 384.0864; found: 384.0860 .

General procedure for the photopromoted goldcatalyzed cross double arylation reaction of TMS- 
(ethynyl)azidobenzene 4a-Si with diazonium salts 2. Preparation of crossed-3-methoxy-2,3-diaryl-3H-indoles 6aag-6aeb. In a Schlenk tube in the absence of light at -78 ${ }^{\circ} \mathrm{C}$ under argon atmosphere, $\mathrm{Ph}_{3} \mathrm{PAuCl}(5 \mathrm{~mol} \%)$ and $\left[\mathrm{Ru}(\mathrm{bpy})_{3]}\left(\mathrm{PF}_{6}\right)_{2}(2.5 \mathrm{~mol} \%)\right.$ were sequentially added to a solution of the first arene diazonium salt 2 (1.5 equiv) in a mixture of $\mathrm{MeOH} / \mathrm{MeCN}$ (3:1, $4.0 \mathrm{~mL}$ ). Then, a solution of the 2-[(trimethylsilyl)ethynyl]azidobenzene 4a-Si (1.0 $\mathrm{mmol})$ in $\mathrm{MeOH} / \mathrm{MeCN}$ (3:1, $1.5 \mathrm{~mL}$ ) was added dropwise and the reaction was stirred at $-78{ }^{\circ} \mathrm{C}$ for $5 \mathrm{~min}$. The reaction mixture was then warmed to room temperature and stirred under irradiation from visible light source ( $21 \mathrm{~W}$ fluorescent light bulb installed in a tool box). After disappearance of the starting material (TLC, 20-30 min), $\mathrm{Ph}_{3} \mathrm{PAuCl}$ (5 mol \%) and a solution of the second arene diazonium salt 2 (6.0 equiv) in a mixture of $\mathrm{MeOH} / \mathrm{MeCN}(3: 1,2.5 \mathrm{~mL}$ ) were sequentially added. The resulting reaction mixture was stirred at rt under irradiation from visible light source (21 W fluorescent light bulb installed in a tool box). After disappearance of the starting material (TLC), the reaction mixture was concentrated under reduced pressure. Chromatography of the residue using hexanes/ethyl acetate or hexanes/toluene mixtures gave analytically pure compounds. The addition of $\mathrm{Et}_{3} \mathrm{~N}$ (2\%) to the eluent was neccesary for the purification of acid sensitive $3 H$-indoles 6 . Spectroscopic and analytical data for pure forms of crossed adducts 6 follow.

3-Methoxy-2,3-diaryl-3H-indole 6abe. From 30 mg (0.14 mmol) of TMS-azide 4a-Si, and after chromatography of the residue using hexanes/Et ${ }_{2} \mathrm{O}(97: 3)$ containing $\mathrm{NEt}_{3}$ (2\%) as eluent, gave compound 6abe (35 mg, 61\%) as a yellow oil; ${ }^{1} \mathrm{H}$ NMR (300 MHz, $\left.\mathrm{CDCl}_{3}, 25^{\circ} \mathrm{C}\right) \delta: 7.99\left(\mathrm{~m}, 2 \mathrm{H}, 2 \mathrm{CH}^{\mathrm{Ar}}\right)$, 7.59 (d, $1 \mathrm{H}, J=7.7 \mathrm{~Hz}, \mathrm{CH}^{\mathrm{Ar}}$ ), 7.42 (m, 2H, 2CH ${ }^{\mathrm{Ar}}$ ), 7.32 (td, $1 \mathrm{H}, J=7.6,1.3 \mathrm{~Hz}, \mathrm{CH}^{\mathrm{Ar}}$ ), 7.15 (m, 5H, 5CH $\mathrm{CH}^{\mathrm{Ar}}$ ), 7.02 (d, $1 \mathrm{H}, J=7.3 \mathrm{~Hz}, \mathrm{CH}^{\mathrm{Ar}}$ ), 2.98 (s, $\left.3 \mathrm{H}, \mathrm{OCH}_{3}\right) ;{ }^{13} \mathrm{C}$ NMR $\left(75 \mathrm{MHz}, \mathrm{CDCl}_{3}, 25^{\circ} \mathrm{C}\right) \delta: 177.3(\mathrm{~N}=\mathrm{C}), 153.3\left(C^{\mathrm{Ar}-\mathrm{q}}\right)$, $139.8\left(C^{\mathrm{Ar}-\mathrm{q}}\right), 137.7\left(C^{\mathrm{Ar}-\mathrm{q}}\right), 133.5\left(C^{\mathrm{Ar}-\mathrm{q}}\right), 131.9\left(2 \mathrm{CH}^{\mathrm{Ar}}\right)$, $130.1\left(\mathrm{CH}^{\mathrm{Ar}}\right), 130.0\left(C^{\mathrm{Ar}-\mathrm{q}}\right), 129.9\left(2 \mathrm{CH}^{\mathrm{Ar}}\right), 129.0\left(\mathrm{CH}^{\mathrm{Ar}}\right)$, $127.2\left(\mathrm{CH}^{\mathrm{Ar}}\right), 126.3\left(C^{\mathrm{Ar}-\mathrm{q}}\right), 125.9\left(2 \mathrm{CH}^{\mathrm{Ar}}\right), 123.4\left(\mathrm{CH}^{\mathrm{Ar}}\right)$, 121.6 $\left(\mathrm{CH}^{\mathrm{Ar}}\right)$, $92.9\left(\mathrm{OC}^{\mathrm{q}}\right), 52.6\left(\mathrm{OCH}_{3}\right)$; IR $\left(\mathrm{CHCl}_{3}, \mathrm{~cm}^{-1}\right)$ : v $1693(\mathrm{~N}=\mathrm{C}), 1085$ (C-O); HRMS (ES): calcd for $\mathrm{C}_{21} \mathrm{H}_{16} \mathrm{BrClNO}\left[M+\mathrm{H}^{+}\right.$: 412.0098; found: 412.0115 .

3-Methoxy-2,3-diaryl-3H-indole 6aeb. From 30 mg (0.14 mmol) of TMS-azide $4 \mathbf{a}-\mathbf{S i}$, and after chromatography of the residue using hexanes/ $\mathrm{Et}_{2} \mathrm{O}(97: 3)$ containing $\mathrm{NEt}_{3}$ (2\%) as eluent, gave compound 6aeb (33 mg, 57\%) as a yellow oil; ${ }^{1} \mathrm{H}$ NMR (300 MHz, $\left.\mathrm{CDCl}_{3}, 25{ }^{\circ} \mathrm{C}\right) \delta: 8.04\left(\mathrm{~m}, 2 \mathrm{H}, 2 \mathrm{CH}^{\mathrm{Ar}}\right.$ ), 7.38 (d, $1 \mathrm{H}, J=7.7 \mathrm{~Hz}, \mathrm{CH}^{\mathrm{Ar}}$ ), 7.38 (m, 5H, 5CH ${ }^{\mathrm{Ar}}$ ), 7.21 (m, 3H, 3CH ${ }^{\mathrm{Ar}}$ ), 7.11 (d, $1 \mathrm{H}, J=7.3 \mathrm{~Hz}, \mathrm{CH}^{\mathrm{Ar}}$ ), 3.07 (s, 3H, $\left.\mathrm{OCH}_{3}\right) ;{ }^{13} \mathrm{C} \mathrm{NMR}\left(75 \mathrm{MHz}, \mathrm{CDCl}_{3}, 25^{\circ} \mathrm{C}\right) \delta: 177.1(\mathrm{~N}=\mathrm{C})$, $153.3\left(C^{\text {Ar-q }}\right), 139.7\left(C^{\text {Ar-q }}\right), 138.3\left(C^{\text {Ar-q }}\right), 137.7\left(C^{\text {Ar-q }}\right)$, $131.9\left(2 \mathrm{CH}^{\mathrm{Ar}}\right), 130.1\left(\mathrm{CH}^{\mathrm{Ar}}\right), 129.7\left(2 \mathrm{CH}^{\mathrm{Ar}}\right), 129.6\left(C^{\mathrm{Ar}-\mathrm{q}}\right)$, $128.9\left(2 \mathrm{CH}^{\mathrm{Ar}}\right), 127.1\left(\mathrm{CH}^{\mathrm{Ar}}\right), 126.2\left(2 \mathrm{CH}^{\mathrm{Ar}}\right), 123.4\left(\mathrm{CH}^{\mathrm{Ar}}\right)$, $121.6\left(C^{\mathrm{Ar}-\mathrm{q}}\right), 121.5\left(\mathrm{CH}^{\mathrm{Ar}}\right), 92.9\left(\mathrm{OC}^{\mathrm{q}}\right), 52.6\left(\mathrm{OCH}_{3}\right)$; IR $\left(\mathrm{CHCl}_{3}, \mathrm{~cm}^{-1}\right): v 1726(\mathrm{~N}=\mathrm{C}), 1087$ (C-O); HRMS (ES): calcd for $\mathrm{C}_{21} \mathrm{H}_{16} \mathrm{BrClNO}\left[M+\mathrm{H}^{+}\right.$: 412.0098; found: 412.0087.

General procedure for the base-catalyzed rearrangement reaction of 3-methoxy-2,3-diaryl-3H- indoles 6. Preparation of 2,3-diaryl-1H-indoles 5 . A solution of the corresponding $3 \mathrm{H}$-indole $\mathbf{6}(1 \mathrm{mmol})$ in ethanolic potash (115 mL, KOH $1 \mathrm{M}$ in EtOH) was stirred under microwave heating $\left(105{ }^{\circ} \mathrm{C}\right)$ until the complete disappearance of the starting material (TLC, typically $12 \mathrm{~h}$ ). After this time, the reaction was cooled down to $0{ }^{\circ} \mathrm{C}$ and neutralized with $\mathrm{HCl}(3 \mathrm{M})$ until $\mathrm{pH}$ 7. The aqueous phase was extracted with EtOAc $(3 \times 20 \mathrm{~mL})$, the organic phases combined, dried over $\mathrm{MgSO}_{4}$ and the solvent removed by distillation under reduced pressure. Chromatography of the residue eluting with ethyl acetate/hexanes mixtures gave analytically pure adducts 5 .

2,3-Diaryl-1H-indole 5ab. From $20 \mathrm{mg}(0.043 \mathrm{mmol})$ of $3 \mathrm{H}$-indole $\mathbf{6 a b}$, and after chromatography of the residue using hexanes/DCM (8:2) as eluent, gave compound 5ab (13 mg, 71\%) as a colorless solid; m.p. $175-177{ }^{\circ} \mathrm{C} ;{ }^{1} \mathrm{H}$ NMR (300 MHz, $\left.\mathrm{CDCl}_{3}, 25^{\circ} \mathrm{C}\right) \delta: 8.21$ (s, 1H, NH), 7.62 (d, $\left.1 \mathrm{H}, J=7.9 \mathrm{~Hz}, \mathrm{CH}^{\mathrm{Ar}}\right), 7.47\left(\mathrm{~m}, 5 \mathrm{H}, 5 \mathrm{CH}^{\mathrm{Ar}}\right), 7.27$ (m, $5 \mathrm{H}, 5 \mathrm{CH}^{\mathrm{Ar}}$ ), $7.17\left(\mathrm{~m}, 1 \mathrm{H}, \mathrm{CH}^{\mathrm{Ar}}\right) ;{ }^{13} \mathrm{C} \mathrm{NMR}\left(75 \mathrm{MHz} \mathrm{CDCl}_{3}\right.$, $\left.25{ }^{\circ} \mathrm{C}\right) \delta: 135.9\left(C^{\mathrm{Ar}-\mathrm{q}}\right), 133.7\left(C^{\mathrm{Ar}-\mathrm{q}}\right), 133.0\left(C^{\mathrm{Ar}-\mathrm{q}}\right), 132.0$ $\left(2 \mathrm{CH}^{\mathrm{Ar}}\right), 131.8\left(2 \mathrm{CH}^{\mathrm{Ar}}\right), 131.6\left(2 \mathrm{CH}^{\mathrm{Ar}}\right), 131.2\left(C^{\mathrm{Ar}-\mathrm{q}}\right), 129.6$ $\left(2 \mathrm{CH}^{\mathrm{Ar}}\right), 128.3\left(C^{\mathrm{Ar}-\mathrm{q}}\right), 123.2\left(\mathrm{CH}^{\mathrm{Ar}}\right), 122.1\left(C^{\mathrm{Ar}-\mathrm{q}}\right), 120.8$ $\left(\mathrm{CH}^{\mathrm{Ar}}\right), 120.4\left(C^{\mathrm{Ar}-\mathrm{q}}\right), 119.4\left(\mathrm{CH}^{\mathrm{Ar}}\right), 114.3\left(C^{\mathrm{Ar}-\mathrm{q}}\right), 111.0$ $\left(\mathrm{CH}^{\mathrm{Ar}}\right)$; IR $\left(\mathrm{CHCl}_{3}, \mathrm{~cm}^{-1}\right)$ : v $3417(\mathrm{~N}-\mathrm{H})$; HRMS (ES): calcd for $\mathrm{C}_{20} \mathrm{H}_{14} \mathrm{Br}_{2} \mathrm{~N}[M+\mathrm{H}]^{+}$: 425.9487; found: 425.9484 .

General procedure for the acid-catalyzed rearrangement reaction of 3-methoxy-2,3-diaryl-3Hindoles 6. Preparation of 2-oxindoles 8 . A solution of the corresponding $3 \mathrm{H}$-indole $\mathbf{6}$ ( $1 \mathrm{mmol})$ in ethanolic sulphuric acid (115 mL, $\mathrm{H}_{2} \mathrm{SO}_{4} 0.4 \mathrm{M}$ in EtOH) was stirred under microwave heating $\left(125{ }^{\circ} \mathrm{C}\right)$ until the complete disappearance of the starting material (TLC, typically $12 \mathrm{~h}$ ). After this time, the reaction was cooled down to $0{ }^{\circ} \mathrm{C}$ and neutralized with $\mathrm{NaHCO}_{3}$ (aqueous saturated solution) until $\mathrm{pH}$ 7. The aqueous phase was extracted with EtOAc $(3 \times 10$ $\mathrm{mL})$, the organic phases combined, dried over $\mathrm{MgSO}_{4}$ and the solvent removed by distillation under reduced pressure. Chromatography of the residue eluting with ethyl acetate/hexanes mixtures gave analytically pure adducts $\mathbf{8}$.

3,3-Diaryl-2-oxindole 8ab. From $20 \mathrm{mg}$ (0.043 mmol) of $3 \mathrm{H}$-indole $\mathbf{6 a b}$, and after chromatography of the residue using hexanes/AcOEt $(97: 3 \rightarrow 80: 20)$ as eluent, gave compound 8ab (14 mg, 72\%) as a colorless oil; ${ }^{1} \mathrm{H}$ NMR $\left(500 \mathrm{MHz}, \mathrm{CDCl}_{3}, 25^{\circ} \mathrm{C}\right) \delta: 8.48$ (s, $\left.1 \mathrm{H}, \mathrm{NH}\right), 7.44$ (m, 4H, $\left.4 \mathrm{CH}^{\mathrm{Ar}}\right), 7.28\left(\mathrm{t}, 1 \mathrm{H}, J=7.6 \mathrm{~Hz}, \mathrm{CH}^{\mathrm{Ar}}\right), 7.16\left(\mathrm{~m}, 5 \mathrm{H}, 5 \mathrm{CH}^{\mathrm{Ar}}\right)$, 7.09 (t, $\left.1 \mathrm{H}, J=7.6 \mathrm{~Hz}, \mathrm{CH}^{\mathrm{Ar}}\right), 6.98(\mathrm{~d}, 1 \mathrm{H}, J=7.8 \mathrm{~Hz}$, $\left.\mathrm{CH}^{\mathrm{Ar}}\right) .{ }^{13} \mathrm{C} \mathrm{NMR}\left(125 \mathrm{MHz}, \mathrm{CDCl}_{3}, 25^{\circ} \mathrm{C}\right) \delta: 178.8(\mathrm{C}=\mathrm{O})$, $140.2\left(2 C^{\mathrm{Ar}-\mathrm{q}}\right), 139.9\left(C^{\mathrm{Ar}-\mathrm{q}}\right), 132.4\left(C^{\mathrm{Ar}-\mathrm{q}}\right), 131.7\left(4 \mathrm{CH}^{\mathrm{Ar}}\right)$, $130.1\left(4 \mathrm{CH}^{\mathrm{Ar}}\right), 128.8\left(\mathrm{CH}^{\mathrm{Ar}}\right), 126.1\left(\mathrm{CH}^{\mathrm{Ar}}\right), 123.2\left(\mathrm{CH}^{\mathrm{Ar}}\right)$, $121.9\left(2 C^{\mathrm{Ar}-\mathrm{q}}\right), 110.5\left(\mathrm{CH}^{\mathrm{Ar}}\right), 61.0\left(C^{\mathrm{q}}-\mathrm{C}=\mathrm{O}\right)$. IR $\left(\mathrm{CHCl}_{3}\right.$, $\left.\mathrm{cm}^{-1}\right)$ : v. $3222(\mathrm{~N}-\mathrm{H}), 1711(\mathrm{C}=\mathrm{O})$; HRMS (ES): calcd for $\mathrm{C}_{20} \mathrm{H}_{14} \mathrm{Br}_{2} \mathrm{NO}[M+\mathrm{H}]^{+}$: 441.9437; found: 441.9446 .

General procedure for the acid-catalyzed rearrangement reaction of 3-methoxy-2,3-diaryl-3Hindoles 6. Preparation of 3-oxindolines 9. A solution of the corresponding $3 \mathrm{H}$-indole $\mathbf{6}(1 \mathrm{mmol})$ in ethanolic sulphuric acid (115 mL, $\mathrm{H}_{2} \mathrm{SO}_{4} 0.2 \mathrm{M}$ in EtOH) was stirred under microwave heating $\left(95{ }^{\circ} \mathrm{C}\right)$ until the complete 
disappearance of the starting material (TLC, typically $12 \mathrm{~h}$ ). After this time, the reaction was cooled down to $0{ }^{\circ} \mathrm{C}$ and neutralized with $\mathrm{NaHCO}_{3}$ (aqueous saturated solution) until $\mathrm{pH}$ 7. The aqueous phase was extracted with EtOAc $(3 \times 10$ $\mathrm{mL}$ ), the organic phases combined, dried over $\mathrm{MgSO}_{4}$ and the solvent removed by distillation under reduced pressure. Chromatography of the residue eluting with ethyl acetate/hexanes mixtures gave analytically pure adducts $\mathbf{9}$ along with compounds $\mathbf{8}$ as minor components.

2,2-Diaryl-3-oxindoline 9ab. From $20 \mathrm{mg}$ (0.043 mmol) of $3 \mathrm{H}$-indole $\mathbf{6 a b}$, and after chromatography of the residue using hexanes:AcOEt (97:3) as eluent, gave compound 9ab (10 mg, 51\%) yellow oil; ${ }^{1} \mathrm{H}$ NMR (500 MHz, $\mathrm{CDCl}_{3}, 25$ $\left.{ }^{\circ} \mathrm{C}\right) \delta: 7.66\left(\mathrm{~d}, 1 \mathrm{H}, J=7.7 \mathrm{~Hz}, \mathrm{CH}^{\mathrm{Ar}}\right), 7.53(\mathrm{t}, 1 \mathrm{H}, J=7.7$ $\mathrm{Hz}, \mathrm{CH}^{\mathrm{Ar}}$ ), 7.46 (m, 4H, 4CH ${ }^{\mathrm{Ar}}$ ), 7.25 (m, 4H, 4CH $\left.{ }^{\mathrm{Ar}}\right), 6.94$ $\left(\mathrm{m}, 2 \mathrm{H}, 2 \mathrm{CH}^{\mathrm{Ar}}\right), 5.09$ (s, 1H, NH). ${ }^{13} \mathrm{C}$ NMR $(125 \mathrm{MHz}$, $\left.\mathrm{CDCl}_{3}, 25^{\circ} \mathrm{C}\right) \delta: 199.8(\mathrm{C}=\mathrm{O}), 159.9\left(C^{\mathrm{Ar}-\mathrm{q}}\right), 139.7\left(2 C^{\mathrm{Ar}-\mathrm{q}}\right)$, $138.0\left(\mathrm{CH}^{\mathrm{Ar}}\right), 131.8\left(4 \mathrm{CH}^{\mathrm{Ar}}\right), 129.1\left(4 \mathrm{CH}^{\mathrm{Ar}}\right), 125.6\left(\mathrm{CH}^{\mathrm{Ar}}\right)$, $122.4\left(2 C^{\mathrm{Ar}-\mathrm{q}}\right), 120.3\left(\mathrm{CH}^{\mathrm{Ar}}\right), 119.8\left(C^{\mathrm{Ar}-\mathrm{q}}\right), 112.7\left(\mathrm{CH}^{\mathrm{Ar}}\right)$, $74.0\left(\mathrm{NC}^{\mathrm{q}}\right)$. IR $\left(\mathrm{CHCl}_{3}, \mathrm{~cm}^{-1}\right): \quad v 3450(\mathrm{~N}-\mathrm{H}), 1686$ (C=O). HRMS (ES): calcd for $\mathrm{C}_{20} \mathrm{H}_{14} \mathrm{Br}_{2} \mathrm{NO}[M+\mathrm{H}]^{+}$: 441.9437; found: 441.9453.

General procedure for the photopromoted goldcatalyzed monoarylation reaction of aryl-terminated alkynones 14-Ph or 16-Ph with diazonium salts 2 . Preparation of 3-aryl-flavones 15 and 3-arylthioflavones 17. In a Schlenk tube in the absence of light at $-78{ }^{\circ} \mathrm{C}$ under argon atmosphere, $\mathrm{Ph}_{3} \mathrm{PAuCl}(10 \mathrm{~mol} \%)$ and $\left[\mathrm{Ru}(\mathrm{bpy})_{3]}\left(\mathrm{PF}_{6}\right)_{2}\right.$ (2.5 mol \%) were sequentially added to a solution of the corresponding arene diazonium salt 2 (3.0 equiv) in a mixture of $\mathrm{MeOH} / \mathrm{MeCN}$ (3:1, $3 \mathrm{~mL}$ ). Then, a solution of the appropriate aryl-terminated alkynone 14-Ph or 16-Ph $(1.0 \mathrm{mmol})$ in $\mathrm{MeOH} / \mathrm{MeCN}(3: 1,2.5 \mathrm{~mL})$ was added dropwise and the reaction was stirred at $-78^{\circ} \mathrm{C}$ for 5 min. The reaction mixture was then warmed to room temperature and stirred under irradiation from visible light source (21 W fluorescent light bulb installed in a tool box). After disappearance of the starting material (TLC), the reaction mixture was concentrated under reduced pressure. Chromatography of the residue using hexanes/ethyl acetate or hexanes/toluene mixtures gave analytically pure compounds. Spectroscopic and analytical data for pure forms of compounds $\mathbf{1 5}$ and $\mathbf{1 7}$ follow.

3-Aryl-flavone 15c. From $21 \mathrm{mg}(0.10 \mathrm{mmol})$ of arylterminated alkynone 14-Ph, and after chromatography of the residue using hexanes/ethyl acetate (95:5) as eluent, gave compound 15c (24 mg, 66\%) as a colorless solid; m.p. 127-129 ${ }^{\circ} \mathrm{C}$; ${ }^{1} \mathrm{H}$ NMR $\left(300 \mathrm{MHz}, \mathrm{CDCl}_{3}, 25^{\circ} \mathrm{C}\right.$ ) $\delta: 8.32$ (dd, $\left.1 \mathrm{H}, J=7.6 \mathrm{~Hz}, J=1.5 \mathrm{~Hz}, \mathrm{CH}^{\mathrm{Ar}}\right), 8.00(\mathrm{~d}, 2 \mathrm{H}, J=8.3 \mathrm{~Hz}$, $\left.2 \mathrm{CH}^{\mathrm{Ar}}\right), 7.77-7.71\left(\mathrm{~m}, 1 \mathrm{H}, 1 \mathrm{CH}^{\mathrm{Ar}}\right), 7.56(\mathrm{~d}, 1 \mathrm{H}, J=8.6 \mathrm{~Hz}$, $\left.1 \mathrm{CH}^{\mathrm{Ar}}\right)$, 7.49-7.27 (m, 8H, 8CH $\left.{ }^{\mathrm{Ar}}\right), 4.38(\mathrm{q}, 2 \mathrm{H}, J=6.5 \mathrm{~Hz}$, $\left.\mathrm{CH}_{2}\right), 1.40\left(\mathrm{t}, 3 \mathrm{H}, J=6.5 \mathrm{~Hz}, \mathrm{CH}_{3}\right) ;{ }^{13} \mathrm{C} \mathrm{NMR}(75 \mathrm{MHz}$, $\left.\mathrm{CDCl}_{3}, 25^{\circ} \mathrm{C}\right) \delta: 176.8(\mathrm{C}=\mathrm{O}), 166.4(\mathrm{C}=\mathrm{O}), 161.8(\mathrm{C}=\mathrm{C})$, $155.9\left(C^{\mathrm{Ar}-\mathrm{q}}\right), 137.8\left(C^{\mathrm{Ar}-\mathrm{q}}\right), 133.8\left(\mathrm{CH}^{\mathrm{Ar}}\right), 132.8\left(C^{\mathrm{Ar}-\mathrm{q}}\right)$, $131.3\left(2 \mathrm{CH}^{\mathrm{Ar}}\right), 130.3\left(\mathrm{CH}^{\mathrm{Ar}}\right), 129.5\left(2 \mathrm{CH}^{\mathrm{Ar}}\right), 129.4\left(C^{\mathrm{Ar}-\mathrm{q}}\right)$, $129.3\left(2 \mathrm{CH}^{\mathrm{Ar}}\right), 128.2\left(2 \mathrm{CH}^{\mathrm{Ar}}\right), 126.3\left(\mathrm{CH}^{\mathrm{Ar}}\right), 125.2\left(\mathrm{CH}^{\mathrm{Ar}}\right)$, $123.3\left(C^{\mathrm{Ar}-\mathrm{q}}\right), 122.1\left(C^{\mathrm{Ar}-\mathrm{q}}\right), 118.0\left(\mathrm{CH}^{\mathrm{Ar}}\right), 60.9\left(\mathrm{CH}_{2}\right), 14.3$ $\left(\mathrm{CH}_{3}\right)$; IR $\left(\mathrm{CHCl}_{3}, \mathrm{~cm}^{-1}\right): v 1712(\mathrm{C}=\mathrm{O}), 1639(\mathrm{C}=\mathrm{O})$,
1270; HRMS (ES): calcd for $\mathrm{C}_{24} \mathrm{H}_{19} \mathrm{O}_{4}[M+\mathrm{H}]^{+}$: 371.1278 ; found: 371.12826 .

3-Aryl-flavone 15f. From $21 \mathrm{mg}(0.10 \mathrm{mmol})$ of arylterminated alkynone 14-Ph, and after chromatography of the residue using hexanes/ethyl acetate (95:5) as eluent, gave compound $15 f$ ( $25 \mathrm{mg}, 69 \%$ ) as a colorless solid; m.p. 144-145 ${ }^{\circ} \mathrm{C}$; ${ }^{1} \mathrm{H}$ NMR (500 MHz, $\mathrm{CDCl}_{3}, 25^{\circ} \mathrm{C}$ ) $\delta: 8.31$ (dd, $\left.1 \mathrm{H}, J=7.6 \mathrm{~Hz}, J=1.5 \mathrm{~Hz}, \mathrm{CH}^{\mathrm{Ar}}\right), 7.76-7.73(\mathrm{~m}, 2 \mathrm{H}$, $\left.2 \mathrm{CH}^{\mathrm{Ar}}\right), 7.57\left(\mathrm{~d}, 1 \mathrm{H}, J=8.6 \mathrm{~Hz}, \mathrm{CH}^{\mathrm{Ar}}\right), 7.49-7.46(\mathrm{~m}, 1 \mathrm{H}$, $\left.1 \mathrm{CH}^{\mathrm{Ar}}\right)$, 7.40-7.31 (m, 5H, 5CH $\left.{ }^{\mathrm{Ar}}\right) ;{ }^{13} \mathrm{C}$ NMR (125 MHz, $\left.\mathrm{CDCl}_{3}, 25^{\circ} \mathrm{C}\right) \delta: 176.8(\mathrm{C}=\mathrm{O}), 162.1(C=\mathrm{C}), 159.0\left(C^{\mathrm{Ar}-\mathrm{q}}\right)$, $136.8\left(C^{\mathrm{Ar}-\mathrm{q}}\right), 134.0\left(\mathrm{CH}^{\mathrm{Ar}}\right), 131.7\left(2 \mathrm{CH}^{\mathrm{Ar}}\right), 130.5\left(\mathrm{CH}^{\mathrm{Ar}}\right)$, $129.5\left(2 \mathrm{CH}^{\mathrm{Ar}}\right), 129.6\left(\mathrm{q}, J_{C F}=32.0 \mathrm{~Hz}, C^{\mathrm{Ar}-\mathrm{q}}\right), 128.3$ $\left(2 \mathrm{CH}^{\mathrm{Ar}}\right), 126.5\left(\mathrm{CH}^{\mathrm{Ar}}\right), 125.4\left(\mathrm{CH}^{\mathrm{Ar}}\right), 125.4\left(\mathrm{q}, J_{C F}=4.3\right.$ $\left.\mathrm{Hz}, 2 \mathrm{CH}^{\mathrm{Ar}}\right), 124.1$ (q, $\left.J_{C F}=272.0 \mathrm{~Hz}, \mathrm{CF}_{3}\right), 123.3\left(C^{\mathrm{Ar}-\mathrm{q}}\right)$, $121.7\left(C^{\mathrm{Ar}-\mathrm{q}}\right), 118.0\left(\mathrm{CH}^{\mathrm{Ar}}\right) ;{ }^{19} \mathrm{~F}$ NMR $\left(282 \mathrm{MHz}, \mathrm{CDCl}_{3}, 25\right.$ $\left.{ }^{\circ} \mathrm{C}\right):-62.9$ (s, 3F, $\left.\mathrm{CF}_{3}\right)$; IR $\left(\mathrm{CHCl}_{3}, \mathrm{~cm}^{-1}\right): v 1638(\mathrm{C}=\mathrm{O})$, 1377; HRMS (ES): calcd for $\mathrm{C}_{22} \mathrm{H}_{14} \mathrm{~F}_{3} \mathrm{O}_{2}[M+\mathrm{H}]^{+}$: 367.0904; found: 367.0947.

3-Aryl-thioflavone $17 \mathbf{b}$. From $35 \mathrm{mg}(0.14 \mathrm{mmol})$ of arylterminated alkynone 16-Ph, and after chromatography of the residue using hexanes/ethyl acetate (95:5) as eluent, gave compound $\mathbf{1 7 b}$ (33 mg, 61\%) as a colorless solid; m.p. 155-157 ${ }^{\circ} \mathrm{C} ;{ }^{1} \mathrm{H}$ NMR (300 MHz, $\left.\mathrm{CDCl}_{3}, 25^{\circ} \mathrm{C}\right) \delta: 8.50(\mathrm{~d}$, $\left.1 \mathrm{H}, J=7.7 \mathrm{~Hz}, \mathrm{CH}^{\mathrm{Ar}}\right), 7.52\left(\mathrm{~m}, 3 \mathrm{H}, 3 \mathrm{CH}^{\mathrm{Ar}}\right), 7.20(\mathrm{~m}, 7 \mathrm{H}$, $\left.7 \mathrm{CH}^{\mathrm{Ar}}\right), 6.91\left(\mathrm{~m}, 2 \mathrm{H}, 2 \mathrm{CH}^{\mathrm{Ar}}\right) ;{ }^{13} \mathrm{C} \mathrm{NMR}\left(75 \mathrm{MHz} \mathrm{CDCl}_{3}\right.$, $\left.25^{\circ} \mathrm{C}\right) \delta: 179.2(\mathrm{C}=\mathrm{O}), 150.8(C=\mathrm{C}), 137.2\left(C^{\mathrm{Ar}-\mathrm{q}}\right), 136.6$ $\left(C^{\mathrm{Ar}-\mathrm{q}}\right), 134.5\left(C^{\mathrm{Ar}-\mathrm{q}}\right), 134.4(\mathrm{C}=C), 132.8\left(2 \mathrm{CH}^{\mathrm{Ar}}\right), 131.6$ $\left(\mathrm{CH}^{\mathrm{Ar}}\right), 131.1\left(C^{\mathrm{Ar}-\mathrm{q}}\right), 130.9\left(2 \mathrm{CH}^{\mathrm{Ar}}\right), 129.5\left(\mathrm{CH}^{\mathrm{Ar}}\right), 129.3$ $\left(\mathrm{CH}^{\mathrm{Ar}}\right), 129.2\left(2 \mathrm{CH}^{\mathrm{Ar}}\right), 128.5\left(2 \mathrm{CH}^{\mathrm{Ar}}\right), 127.8\left(\mathrm{CH}^{\mathrm{Ar}}\right), 125.8$ $\left(\mathrm{CH}^{\mathrm{Ar}}\right), 121.4\left(\mathrm{C}^{\mathrm{Ar}-\mathrm{q}}\right)$; IR $\left(\mathrm{CHCl}_{3}, \mathrm{~cm}^{-1}\right): v 1617(\mathrm{C}=\mathrm{O})$, 1589 (C=C); HRMS (ES): calcd for $\mathrm{C}_{21} \mathrm{H}_{14} \mathrm{BrOS}[M+\mathrm{H}]^{+}$: 392.9943; found: 392.9935 .

3-Aryl-thioflavone 17e. From $31 \mathrm{mg}(0.12 \mathrm{mmol})$ of arylterminated alkynone 16-Ph, and after chromatography of the residue using hexanes/ethyl acetate (95:5) as eluent, gave compound 17e (28 mg, 68\%) as a colorless solid; m.p. 154-156 ${ }^{\circ} \mathrm{C} ;{ }^{1} \mathrm{H}$ NMR (500 MHz, $\left.\mathrm{CDCl}_{3}, 25{ }^{\circ} \mathrm{C}\right) \delta: 8.58(\mathrm{~d}$, $\left.1 \mathrm{H}, J=7.8 \mathrm{~Hz}, \mathrm{CH}^{\mathrm{Ar}}\right), 7.62\left(\mathrm{~m}, 3 \mathrm{H}, 3 \mathrm{CH}^{\mathrm{Ar}}\right), 7.28(\mathrm{~m}, 3 \mathrm{H}$, $\left.3 \mathrm{CH}^{\mathrm{Ar}}\right), 7.21\left(\mathrm{~m}, 4 \mathrm{H}, 4 \mathrm{CH}^{\mathrm{Ar}}\right), 7.05\left(2 \mathrm{CH}^{\mathrm{Ar}}\right) ;{ }^{13} \mathrm{C} \mathrm{NMR}(125$ $\left.\mathrm{MHz}, \mathrm{CDCl}_{3}, 25^{\circ} \mathrm{C}\right) \delta: 179.3(\mathrm{C}=\mathrm{O}), 150.8(\mathrm{C}=\mathrm{C}), 137.2$ $\left(C^{\mathrm{Ar}-\mathrm{q}}\right), 136.6\left(C^{\mathrm{Ar}-\mathrm{q}}\right), 134.4\left(C^{\mathrm{Ar}-\mathrm{q}}\right), 134.0(\mathrm{C}=C), 133.1\left(C^{\mathrm{Ar}-}\right.$ q), $132.5\left(2 \mathrm{CH}^{\mathrm{Ar}}\right), 131.6\left(\mathrm{CH}^{\mathrm{Ar}}\right), 131.2\left(C^{\mathrm{Ar}-\mathrm{q}}\right), 129.6\left(\mathrm{CH}^{\mathrm{Ar}}\right)$, $129.3\left(\mathrm{CH}^{\mathrm{Ar}}\right), 129.2\left(2 \mathrm{CH}^{\mathrm{Ar}}\right), 128.5\left(2 \mathrm{CH}^{\mathrm{Ar}}\right), 128.0\left(2 \mathrm{CH}^{\mathrm{Ar}}\right)$, $127.8\left(\mathrm{CH}^{\mathrm{Ar}}\right), 125.8\left(\mathrm{CH}^{\mathrm{Ar}}\right)$; IR $\left(\mathrm{CHCl}_{3}, \mathrm{~cm}^{-1}\right): v 1617$ $(\mathrm{C}=\mathrm{O}), 1588$ (C=C); HRMS (ES): calcd for $\mathrm{C}_{21} \mathrm{H}_{13} \mathrm{ClOS}[M$ $+\mathrm{H}^{+}$: 349.0448; found: 349.0457.

\section{Acknowledgements}

Financial support from the MINECO and FEDER (Projects CTQ2015-65060-C2-1-P and CTQ2015-65060-C2-2-P) is gratefully acknowledged. F. H. and C. L.-M. thank UCM and MINECO, respectively, for predoctoral contracts. E. B. thanks MINECO for a postdoctoral contract. We thank Imanol Burgos for preliminary studies.

\section{References}


[1] a) M. Joost, A. Amgoune, D. Bourissou, Angew. Chem. 2015, 127, 15234; Angew. Chem. Int. Ed. 2015, 54, 15022; b) M. Jia, M. Bandini, ACS Catal. 2015, 5, 1638; c) R. Dorel, A. M. Echavarren, Chem. Rev. 2015, 115, 9028; d) Y. Wang, M. E. Muratore, A. M. Echavarren, Chem. Eur. J. 2015, 21, 7332; e) L. Zhang, Acc. Chem. Res. 2014, 47, 877; f) M. Shi, Acc. Chem. Res. 2014, 47, 913; g) A. S. K. Hashmi, Acc. Chem. Res. 2014, 47, 864; h) C. Obradors, A. M. Echavarren, Acc. Chem. Res. 2014, 47, 902; i) W. Yang, A. S. K. Hashmi, Chem. Soc. Rev. 2014, 43, 2941; j) B. Alcaide, P. Almendros, Acc. Chem. Res. 2014, 47, 939; k) L. Fensterbank, M. Malacria, Acc. Chem. Res. 2014, 47, 953.

[2] a) A. J. Cresswell, G. C. Lloyd-Jones, Chem. Eur. J. 2016, 22, 12641; b) L. T. Ball, G. C. Lloyd-Jones, C. A. Russell, J. Am. Chem. Soc. 2014, 136, 254; c) L. T. Ball, G. C. Lloyd-Jones, C. A. Russell, Science 2012, 337, 1644; d) T. de Haro, C. Nevado, Chem. Commun. 2011, 47, 248; e) H. A. Wegner, M. Auzias, Angew. Chem. Int. Ed. 2011, 50, 8236; f) M. N. Hopkinson, A. D. Gee, V. Gouverneur, Chem. Eur. J. 2011, 17, 8248; g) G. Zhang, L. Cui, Y. Wang, L. Zhang, J. Am. Chem. Soc. 2010, 132, 1474; h) W. E. Brenzovich, D. Benítez, A. D. Lackner, H. P. Shunatona, E. Tkatchouk, W. A. Goddard III, F. D. Toste, Angew. Chem. 2010, 122, 5651; Angew. Chem. Int. Ed. 2010, 49, 5519; i) W. Wang, J. Jasinski, G. B. Hammond, B. Xu, Angew. Chem. 2010, 122, 7405; Angew. Chem. Int. Ed. 2010, 49, 7247; j) M. N. Hopkinson, A. Tessier, A. Salisbury, G. T. Giuffredi, L. E. Combettes, A. D. Gee, V. Gouverneur, Chem. Eur. J. 2010, 16, 4739; k) A. Iglesias, K. Muñiz, Chem. Eur. J. 2009, 15, 9346.

[3] a) B. Sahoo, M. N. Hopkinson, F. Glorius, J. Am. Chem. Soc. 2013, 135, 5505; b) X.-Z. Shu, M. Zhang, Y. He, H. Frei, D. F. Toste, J. Am. Chem. Soc. 2014, 136, 5844.

[4] a) M. N. Hopkinson, B. Sahoo, F. Glorius, Adv. Synth. Catal. 2014, 356, 2794; b) D. V. Patil, H. Yun, S. Shin, Adv. Synth. Catal. 2015, 357, 2622; c) A. Tlahuext-Aca, M. N. Hopkinson, R. A. Garza-Sánchez, F. Glorius, Chem. Eur. J. 2016, 22, 5909; d) V. Gauchot, A.-L. Lee, Chem. Commun. 2016, 52, 10163; e) T. Cornilleau, P. Hermange, E. Fouquet, Chem. Commun. 2016, 52, 10040; f) J. Um, H. Yun, S. Shin, Org. Lett. 2016, 18, 484; g) B. Alcaide, P. Almendros, E. Busto, A. Luna, Adv. Synth. Catal. 2016, 358, 1526; h) V. Gauchot, D. R. Sutherland, A.-L. Lee, Chem. Sci. 2017, 8, DOI: 10.1039/C6SC05469B; i) B. Alcaide, P. Almendros, E. Busto, C. Lázaro-Milla, J. Org. Chem. 2017, 82, 2177.

[5] a) G. Revol, T. McCallum, M. Morin, F. Gagosz, L. Barriault, Angew. Chem. 2013, 125, 13584; Angew. Chem. Int. Ed. 2013, 52, 13342; b) S. J. Kaldas, A. Cannillo, T. McCallum, L. Barriault, Org. Lett. 2015, 17, 2864; c) T. McCallum, L. Barriault, Chem. Sci. 2016, 7, 4754; d) J. Xie, S. Shi, T. Zhang, N. Mehrkens, M. Rudolph, A. S. K. Hashmi, Angew. Chem. 2015, 127, 6144; Angew. Chem. Int. Ed. 2015, 54, 6046; e) J. Xie, J. Li, V. Weingand, M. Rudolph, A. S. K. Hashmi, Chem.
Eur. J. 2016, 22, 12646; f) J. Xie, T. Zhang, F. Chen, N. Mehrkens, F. Rominger, M. Rudolph, A. S. K. Hashmi, Angew. Chem. 2016, 128, 2987; Angew. Chem. Int. Ed. 2016, 55, 2934.

[6] After initiation of our efforts, the groups of Fensterbank and Zhu reported the dual gold/photoredox-catalyzed synthesis of 2,3-diarylbenzofurans and 2,3-diaryl- $1 \mathrm{H}$ indoles, respectively: a) Z. Xia, O. Khaled, V. MourièsMansuy, C. Ollivier, L. Fensterbank, J. Org. Chem. 2016, 81, 7182; b) C. H. Qu, S. Zhang, H. Du, C. Zhu, Chem. Commun. 2016, 52, 14400.

[7] For reviews on photoredox gold catalysis, see: a) T. McCallum, S. Rohe, L. Barriault, Synlett 2017, 28, 289; b) M. Zhang, C. Zhu, L.-W. Ye, Synthesis 2017, 49, 1150; c) M. N. Hopkinson, A. Tlahuext-Aca, F. Glorius, Acc. Chem. Res. 2016, 49, 2261.

[8] For the gold-catalyzed C-H arylation of TMSterminated alkynes, see: a) S. Kim, J. Martín-Rojas, F. D. Toste, Chem. Sci. 2016, 7, 85. For the gold-catalyzed C-H arylation of terminal alkynes, see: b) A. TlahuextAca, M. N. Hopkinson, B. Sahoo, F. Glorius, Chem. Sci. 2016, 7, 89; c) R. Cai, M. Lu, E. Y. Aguilera, Y. Xi, N. G. Akhmedov, J. L. Petersen, H. Chen, X. Shi, Angew. Chem. 2015, 127, 8896; Angew. Chem. Int. Ed. 2015, 54, 8772.

[9] For the gold-catalyzed synthesis of 2-aryl 3-alkyl benzothiophenes by intramolecular thiolation/rearrangement of alkynes, see: I. Nakamura, T. Sato, Y. Yamamoto, Angew. Chem. 2006, 118, 4585; Angew. Chem. Int. Ed. 2006, 45, 4473.

[10] For selected reviews, see: a) X.-R. Song, Y.-F. Qiu, X.-Y. Liu, Y.-M. Liang, Org. Biomol. Chem. 2016, 14, 11317; b) S. Brase, C. Gil, K. Knepper, V. Zimmermann, Angew. Chem. 2005, 117, 5320; Angew. Chem. Int. Ed. 2005, 44, 5188; c) D. Huang, G. Yan, Adv. Synth. Catal. 2017, 359, DOI: 10.1002/adsc.201700103.

[11] For gold-catalyzed cyclizations of azidoalkynes, see: a) A. Wetzel, F. Gagosz, Angew. Chem. 2011, 123, 7492; Angew. Chem. Int. Ed. 2011, 50, 7354; b) B. Lu, Y. Luo, L. Liu, L. Ye, Y. Wang, L. Zhang, Angew. Chem. 2011, 123, 8508; Angew. Chem. Int. Ed. 2011, 50, 8358; c) C. R. Reddy, S. A. Panda, A. Ramaraju, J. Org. Chem. 2017, 82, 944.

[12] TMS-(ethynyl)azidobenzenes 4 are readily available from the reaction of TMS-(ethynyl)anilines and $\mathrm{TMSN}_{3}$.

[13] The preparation of the bioactive 3,3-diaryl-2-oxindole moiety is scarcely reported. For a recent report, see: R. Samineni, C. R. C. Bandi, P. Srihari, G. Mehta, Org. Lett. 2016, 18, 6184.

[14] a) Huang, L.; Rudolph, M.; Rominger, F.; Hashmi, A. S. K. Angew. Chem. 2016, 128, 4888; Angew. Chem. Int. Ed. 2016, 55, 4808; b) L. Huang, F. Rominger, M. Rudolph, A. S. K. Hashmi, Chem. Commun. 2016, 52, 6435.

[15] R. Cai, M. Lu, E. Y. Aguilera, Y. Xi, N. G. Akhmedov, J. L. Petersen, H. Chen, X. Shi, Angew. Chem. 2015, 127, 8896; Angew. Chem. Int. Ed. 2015, 54, 8772. 
[16] Experimental procedures as well as full spectroscopic and analytical data for compounds not included in this Experimental Section are described in the Supporting Information. It contains compound characterization data, experimental procedures, and copies of NMR spectra for all new compounds. 


\section{FULL PAPER}

Photopromoted Entry to Benzothiophenes, Benzoselenophenes, $3 \mathrm{H}$-Indoles, Isocoumarins, Benzosultams, and (Thio)Flavones by GoldCatalyzed Arylative Heterocyclization of Alkynes

Adv. Synth. Catal. 2017, 359, Page - Page

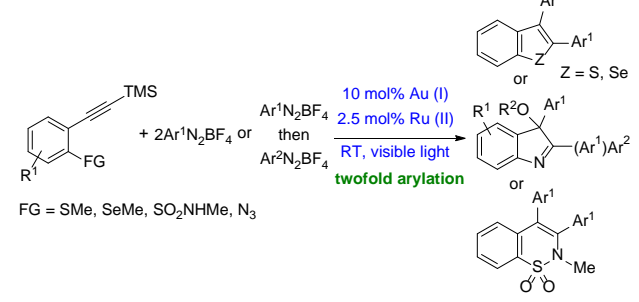

Benito Alcaide,* Pedro Almendros, * Eduardo Busto, Fernando Herrera, Carlos Lázaro-Milla, and

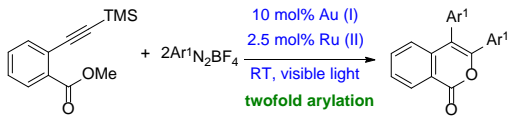
Amparo Luna

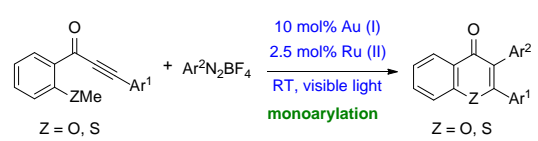

Autores (p.o. de firma): Alcaide, B.; Almendros, P.; Busto, E.; Herrera, F.; Lázaro-Milla, C.; Luna, A.

Título: Photopromoted Entry to Benzothiophenes, Benzoselenophenes, $3 H$-Indoles, Isocoumarins, Benzosultams, and (Thio)Flavones by Gold-Catalyzed Arylative Heterocyclization of Alkynes

Revista: Adv. Synth. Catal. 2017, 359, 2640-2652; DOI: 10.1002/adsc.201700427 